\title{
On the Migration of Jupiter and Saturn: Constraints from Linear Models of Secular Resonant Coupling with the Terrestrial Planets
}

\author{
Craig B. Agnor ${ }^{1}$ and D. N. C. $\operatorname{Lin}^{2,3}$ \\ ${ }^{1}$ Astronomy Unit, School of Physics \& Astronomy, Queen Mary University of London, UK; \\ C.B.Agnor@qmul.ac.uk \\ ${ }^{2}$ Department of Astronomy and Astrophysics, University of California Santa Cruz, CA, USA \\ ${ }^{3}$ Kavli Institute of Astronomy and Astrophysics 8 College of Physics, Peking University, Beijing,
} China

\begin{abstract}
We examine how the late divergent migration of Jupiter and Saturn may have perturbed the terrestrial planets. Using a modified secular model we have identified six secular resonances between the $\nu_{5}$ frequency of Jupiter and Saturn and the four apsidal eigenfrequencies of the terrestrial planets $\left(g_{1-4}\right)$. We derive analytic upper limits on the eccentricity and orbital migration timescale of Jupiter and Saturn when these resonances were encountered to avoid perturbing the eccentricities of the terrestrial planets to values larger than the observed ones. Because of the small amplitudes of the $j=2,3$ terrestrial eigenmodes the $g_{2}-\nu_{5}$ and $g_{3}-\nu_{5}$ resonances provide the strongest constraints on giant planet migration. If Jupiter and Saturn migrated with eccentricities comparable to their present day values, smooth migration with exponential timescales characteristic of planetesimal-driven migration $(\tau \sim 5-10 \mathrm{Myr})$ would have perturbed the eccentricities of the terrestrial planets to values greatly exceeding the observed ones. This excitation may be mitigated if the eccentricity of Jupiter was small during the migration epoch, migration was very rapid (e.g., $\tau \lesssim 0.5 \mathrm{Myr}$ perhaps via planet-planet scattering or instability-driven migration) or the observed small eccentricity amplitudes of the $j=2,3$ terrestrial modes result from low probability cancellation of several large amplitude contributions. Results of orbital integrations show that very short migration timescales $(\tau<0.5 \mathrm{Myr})$, characteristic of instability-driven migration, may also perturb the terrestrial planets' eccentricities by amounts comparable to their observed values. We discuss the implications of these constraints for the relative timing of terrestrial planet formation, giant planet migration, and the origin of the so-called Late Heavy Bombardment of the Moon 3.9 \pm 0.1 Ga ago. We suggest that the simplest way to satisfy these dynamical constraints may be for the bulk of any giant planet migration to be complete in the first $30-100 \mathrm{Myr}$ of solar system history.
\end{abstract}

Subject headings: celestial mechanics - planetary systems: formation - planets and satellites: dynamical evolution and stability - planet-disk interactions - planets and satellites: formation - solar system: formation 


\section{Introduction}

During the last two decades, observational discoveries both external to and within the solar system have provided strong evidence that giant planets experience large-scale orbital migration after their formation. Outside the solar system the peculiar small orbits of the so-called 'Hot Jupiters' and their unlikely in situ formation suggest that these gas giants underwent large-scale, radial migration to their observed short-period orbits (e.g., Lin et al. 1996). At early times ( 1-10Myr) this might be accomplished via interactions with the progenitor gas disk (e.g., Papaloizou et al. 2007). At later times migration may be driven by scattering and redistribution of a planetesimal disk (e.g., Fernandez \& Ip 1984; Murray et al. 1998; Hahn \& Malhotra 1999). In addition, multiplanet systems may suffer orbital instabilities in which giant planets undergo mutual scattering events that may eject planets from the system altogether and leave the surviving ones with large orbital eccentricities and inclinations. Such stochastic evolution can account for the orbital characteristics of many observed extrasolar planets (e.g., Rasio \& Ford 1996; Weidenschilling \& Marzari 1996; Lin \& Ida 1997; Levison et al. 1998).

Closer to home, the resonant structure of the Kuiper Belt testifies to the large-scale radial redistribution of the solar system's giant planets more generally. The eccentric and inclined orbit of Pluto, and the cohort of Plutinos in Neptune's exterior 3:2 mean motion resonance, can be naturally explained by the outward migration of Neptune (Malhotra 1993, 1995; Levison et al. 2008). As the number of solar system characteristics that can be attributed to processes associated with planet migration continues to increase, there is a growing consensus that large-scale planetary migration did occur in the solar system and that many of its dynamical structures were determined during, and represent artifacts of, this epoch (e.g., see reviews by Levison et al. 2007; Chiang et al. 2007).

Due to their large masses and angular momenta, the giant planets are the dominant perturbers of the solar system. Therefore the period of giant planet migration was one in which enormous orbital perturbations swept through the solar system. Clearly the timing and duration of this epoch informs the evolution of the rest of the system.

Because of their hydrogen and helium rich compositions, the formation of Jupiter and Saturn must have been completed during the first few million years of the solar system's history while the gaseous protoplanetary disk still persisted (e.g., see the review by Lissauer \& Stevenson 2007). The accumulation of Uranus and Neptune, is considerably less constrained (Lissauer et al. 1995) and remains an area of active research (e.g., Brvden et al. 2000b; Ward et al. 2001; Thommes et al. 2003; Goldreich et al. 2004; Levison \& Morbidelli 2007). Estimates of the formation timescale of these planets reflect our modest knowledge of their accumulation and span a wide range from $\mathcal{O}\left(10^{6}-10^{9}\right)$ years (Goldreich et al. 2004; Lissauer et al. 1995).

Virtually all models suggested for the accretion of Uranus and Neptune are inefficient at incorporating the mass of an initial planetesimal disk into the ice giants on reasonably short timescales

(e.g., $\lesssim 10 \mathrm{Myr}$ ) and the scattering of these planetesimals may lead to radial spreading and migra- 
tion of the growing planets (Fernandez \& Ip 1984). As a result, disk masses, several times that of the observed planets (i.e., the so-called minimum mass nebula; Weidenschilling 1977) and formation locations well interior to their current orbits are often invoked to account for the accretion of Uranus and Neptune.

However, local concentrations of planet building blocks at planet trapping radii (such as the ice line or the interface between outward and inward migration) may decrease the formation timescale of cores with sufficient mass $\left(\gtrsim 10 M_{\oplus}\right)$ to initiate rapid gas accretion (Kretke \& Lin 2007; Lyra et al. 2010). The formation of Jupiter leads to gap formation in the solar nebula. Near the outer edge of this gap, surface density and pressure maxima (at radii near or beyond Jupiter's 2:1 MMR) also facilitate the accumulation of grains, protoplanetary embryos and the formation of additional giant planet cores (Bryden et al. 2000a , b ). This sequential formation process may provide a scenario for the formation of the gas and ice giant planets before the depletion of the gaseous nebula, with modest, rather than extensive migration, mostly driven by their tidal interaction with both residual disk gas and planetesimals (Ida et al. 2000).

The epoch, mode, and characteristic timescale in which the solar system's giant planets migrated has important implications for the evolution of the planetary system. Giant planet migration models in particle disks can be loosely divided into two classes, based on the principal mode of migration. Planetesimal-driven migration results from the efficient scattering of small bodies by giant planets and is characterized by the smooth radial divergence of planets embedded in a planetesimal disk. Recent models of this process in the solar system suggest that the giant planets' orbits diverge with approximately exponential migration timescales of $\tau \sim a / \dot{a} \simeq 5-20 \mathrm{Myr}$ (e.g., Hahn \& Malhotra 1999; Ida et al. 2000; Gomes et al. 2004).

In contrast to smooth radial planetesimal-driven migration, a global instability among the giant planets, involving close encounters and gravitational scattering between planets, results in large stochastic jumps in the semimajor axes, eccentricities, and inclinations of the planets (Thommes et al. 1999, 2003; Tsiganis et al. 2005; Thommes et al. 2008b; Batygin \& Brown 2010). Because the planets traverse large changes in semimajor axis very quickly, the bulk of planetary migration may be complete in a few million years.

A recent effort to construct a comprehensive model of planetary migration has succeeded in explaining several distinct dynamical characteristics of the solar system as a product of specific giant planet migration histories. The so-called Nice model suggests that the orbits of the giant planets evolved through phases of both planetesimal-driven migration and planet-planet scattering (see Levison et al. 2007, for a review). In initial versions of this model, the instability of the giant planets is initiated by the divergent migration of Jupiter and Saturn across their mutual 2:1 mean motion resonance (Tsiganis et al. 2005). Dynamics associated with the crossing of the 2:1 mean motion resonance (hereafter MMR) play a fundamental role in the Nice model as they are also responsible for facilitating the capture of Jupiter's Trojan asteroids with large orbital inclinations (Morbidelli et al. 2005). 
In the inner solar system, planetary formation may have been completed prior to the time when the giant planets reached their observed orbits. Both dynamical models of terrestrial planet accretion (e.g., Chambers \& Wetherill 1998; Agnor et al. 1999; Chambers 2001; Ravmond et al. 2004; O’Brien et al. 2006; Hansen 2009; Ravmond et al. 2009; Morishima et al. 2010) and cosmochemical evidence (e.g., Kleine et al. 2002; Yin et al. 2002) suggest that terrestrial planet formation was completed in $\sim 30-100 \mathrm{Myr}$.

Uncertainties in the formation timescale of Uranus and Neptune (e.g. ranging from $10^{6}$ to $10^{9}$ yr) combined with estimates of migration timescales $(\tau \sim 5-20 \mathrm{Myr}$ for planetesimal-driven migration or much shorter for planet-planet scattering) suggest that migration of the solar system's giant planets may have taken place before, during, or after terrestrial planet formation. Identifying when and how giant planet migration occurred remains an outstanding problem.

Indeed, the late orbital evolution of the solar system's giant planets has even been invoked to account for cratering events on the Moon, an explanation that necessarily requires fully formed terrestrial planets to bear witness to the epoch of giant planet migration (e.g., Wetherill 1975). The so-called Late Heavy Bombardment of the Moon (hereafter LHB) refers to a period from $~ 3.9 \pm 0.1$ Ga ago when the lunar basins with known ages formed (Tera et al. 1974; see also Hartmann et al. 2000, for a detailed review). The formation of these basins may be attributed to a sharp increase in the cratering rate of the Moon several hundred million years after planet formation was complete. The late orbital evolution of the giant planets has been identified with this dramatic increase in the lunar cratering rate via a variety of dynamical mechanisms including the sweeping of resonances through the asteroid belt due to planetary migration (Strom et al. 2005; Minton \& Malhotra 2009), the late formation of Uranus and Neptune (Levison et al. 2001), orbital instabilities among the giant planets resulting in orbit crossing, and planet-planet gravitational scattering (Thommes et al. 1999, 2002), or an orbital instability resulting from the divergent migration of Jupiter and Saturn across their mutual 2:1 MMR as in the Nice model (Gomes et al. 2005). In each of these models, objects on previously stable orbits are dislodged by the evolution of the giant planets and delivered to Earth crossing orbits. Implicit to each model is the assumption that the terrestrial planets are unaffected by the gross instability and/or changes in the giant planets' orbits which are responsible for destabilizing the LHB impacting population.

Here we examine this assumption in detail. Specifically, we examine how the divergent migration of Jupiter and Saturn from a more compact configuration to their current orbits may perturb the terrestrial planets. If the terrestrial planets witnessed an era of giant planet migration, their present-day orbits offer two main dynamical constraints: (1) the terrestrial planets must remain stable during the epoch of migration and (2) the dynamical structure the terrestrial planets emerging from the migration epoch must be consistent with the observed terrestrial system. Since the solar system's giant planets migrate as a coupled system, any constraints on the migration of Jupiter and Saturn may also be considered as implicit constraints on the formation and migration of Uranus and Neptune. 
We develop linear secular models to identify migration histories of Jupiter and Saturn that strongly couple them to the terrestrial planets via apsidal secular resonances. We adapt models for secular resonant sweeping to predict the excitation of the terrestrial planets as a function of the orbital state of Jupiter and Saturn at the time of resonance and their migration timescale. Finally, we use these models to identify migration histories that are consistent with the observed orbital properties of the terrestrial planets.

This paper is organized in the following fashion. In Section 2, we describe the secular model and its utility for examining planetary migration. In Section 3, we discuss how passage through linear secular resonances excites eccentricities and how these events may be used to constrain giant planet migration histories. In Sections 4 and 5 , we compare the predictions of the linear theory with the results of $N$-body simulations. In Section 6, we evaluate model assumptions, discuss the implications of our prinicipal results, and summarize our findings.

Initial results, including the development of our secular model, the identification of secular resonances, and some numerical simulations were presented in a conference talk (Agnor \& Lin 2007). Subsequently, Brasser et al. (2009) have also examined this problem within the context of the Nice model using $N$-body simulations. Our linear model predictions are in general agreement with, and provide a theoretical framework that accounts for, the results of their simulation results. We compare the two where appropriate.

\section{Secular Evolution of the Planets}

\subsection{Laplace-Lagrange Apsidal Precession}

The apsidal orbital precession of a system of planets can be described using a set of linear firstorder differential equations, whose solution is a system of eigenmodes (see, e.g., Brouwer \& Clemence 1961; Murray \& Dermott 1999). The equations of motion for the evolution of the planets' eccentricities $(e)$ and longitude of perihelia $(\varpi)$ can be constructed from the disturbing function by assuming that terms involving the mean longitudes are small and retaining lowest-order terms in the eccentricity and inclinations. For planet $i$, Lagrange's equations for the eccentricity vector $\left(h_{i}, k_{i}\right)=e_{i}\left(\sin \varpi_{i}, \cos \varpi_{i}\right)$ are

$$
\begin{gathered}
\frac{d h_{i}}{d t}=\sum_{l} A_{i l} k_{l} \\
\frac{d k_{i}}{d t}=-\sum_{l} A_{i l} h_{l},
\end{gathered}
$$

where

$$
\begin{gathered}
A_{i i}=\frac{n_{i} a_{i}}{4} \sum_{l \neq i}\left(\frac{m_{l}}{M_{\odot}+m_{i}}\right) \frac{\alpha}{a_{>}} b_{3 / 2}^{1}+\frac{3 G M_{\odot}}{c^{2} a_{i}} n_{i} \\
A_{i l}=-\frac{n_{i} a_{i}}{4}\left(\frac{m_{l}}{M_{\odot}+m_{i}}\right) \frac{\alpha}{a_{>}} b_{3 / 2}^{2},
\end{gathered}
$$


and $m_{i}, a_{i}$, and $n_{i}$ are a planet's mass, semimajor axis, and mean motion, respectively. The standard terms where $\alpha=a_{<} / a_{>}, a_{>}=\max \left(a_{i}, a_{l}\right), a_{<}=\min \left(a_{i}, a_{l}\right)$, and Laplace coefficients $\left(b_{j}^{s}\right)$ have been used. Post-Newtonian corrections for relativistic precession are included in the diagonal matrix elements where $c$ is the speed of light (see, e.g., Adams \& Laughlin 2006).

Solving these equations for a planetary system is an eigenvalue problem whose eigenfrequencies $\left(g_{j}\right)$ are real and represent the precession frequencies of the modes. The solutions are of the form

$$
\begin{aligned}
& h_{i}=\sum_{j} e_{i j} \sin \left(g_{j} t+\beta_{j}\right) \\
& k_{i}=\sum_{j} e_{i j} \cos \left(g_{j} t+\beta_{j}\right),
\end{aligned}
$$

where the values of modal eccentricity amplitudes $e_{i j}$ and the initial phases of the modes $\beta_{j}$ are constants determined by projecting the system onto some initial state at time $(t=0)$.

\subsection{Eigenmode Amplitudes and the Angular Momentum Deficit}

In our use of this secular model there are two convenient and complementary formulations of the eigenvalue solution, each with its own advantages. The modal eccentricity amplitude $\left(e_{i j}\right)$ solution (above) clearly identifies the orbital variation of each planet $i$ resulting from the coupling with the other planets via mode $j$. As the orbital elements of a system are its principal dynamical coordinates, this formulation is easily interpreted and can be compared with the results of $N$ body simulations (e.g., using Fourier analysis to identify fundamental frequencies and amplitudes). However, this representation of the system's modes tends to mask the structural signature of the eigenvectors and the physical interpretation of a mode's amplitude.

When considering the secular evolution of an isolated system the orbital energies and semimajor axes of the planets are constant and the system angular momentum is conserved. These two constraints may be combined to identify the so-called angular momentum deficit (AMD) as an important integral of a secularly evolving system. The AMD is defined as

$$
\mathrm{AMD}=\sum_{i} m_{i} n_{i} a_{i}^{2}\left(1-\sqrt{1-e_{i}^{2}} \cos I_{i}\right)
$$

where $i$ runs over the planets and $I_{i}$ is a planet's orbital inclination (see, e.g., Laskar 1996). Physically, the AMD represents the angular momentum that must be added to the system to make all the planets' orbits circular and coplanar. Because secular interactions do not alter the semimajor axes of the planets, a system's AMD quantifies the amount of angular momentum that is available for exchange between planets via gravitational interactions and limits the amplitude of eccentricity and inclination oscillations of the planets. 
The linear secular system can also be formulated with the following transformation of variables and matrix elements:

$$
\begin{aligned}
H_{i} & =\sqrt{m_{i} n_{i} a_{i}^{2}} e_{i} \sin \varpi_{i}=\psi_{i} h_{i} \\
K_{i} & =\sqrt{m_{i} n_{i} a_{i}^{2}} e_{i} \cos \varpi_{i}=\psi_{i} k_{i},
\end{aligned}
$$

and $A_{i l} \rightarrow\left(\psi_{i} / \psi_{l}\right) A_{i l}$, where the factor $\psi_{i}$ is the square root of a planet's circular orbital angular momentum (see, e.g., Brouwer \& Clemence 1961).

The system's solution is again expressed as a superposition of the modes

$$
\begin{gathered}
H_{i} \equiv \sum_{j} H_{i j}=\sum_{j} C_{j} v_{i j} \sin \left(g_{j} t+\beta_{j}\right) \\
K_{i} \equiv \sum_{j} K_{i j}=\sum_{j} C_{j} v_{i j} \cos \left(g_{j} t+\beta_{j}\right),
\end{gathered}
$$

where $\mathbf{v}_{j} \equiv\left\{v_{i j}\right\}$ is the $j$ th orthonormal eigenvector and $C_{j}$ its modal amplitude computed from a set of orbital elements and masses. The system quantity $\frac{1}{2} \sum_{i} m_{i} n_{i} a_{i}^{2} e_{i}^{2}=\frac{1}{2}\left(H_{i}^{2}+K_{i}^{2}\right)^{2}=\frac{1}{2} \sum_{j} C_{j}^{2}$ corresponds to the horizontal component of the system's AMD in our secular model. The secular modal amplitudes $\left\{C_{j}\right\}$ are integrals of the system and reveal the fraction of the system AMD partitioned into a single mode. Conversion back and forth between the two formulations is easily accomplished using

$$
e_{i j}=\frac{C_{j} v_{i j}}{\psi_{i}}
$$

As the mass distribution of a planetary system evolves (e.g., due to planetary migration, stellar spin down, dispersal of the gaseous nebula, etc.), the system's eigenfrequencies, eigenvectors, and modal amplitudes become time-dependent. However, if the dynamical tuning of the system is slow with respect to the eigenfrequencies $\left\{g_{j}\right\}$ and the system is not near a secular resonance (i.e., $g_{j}-g_{k}$ is not small), the variation in mode amplitudes tends to oscillate rapidly and average out (see Ward 1981, for a complete development). For slow enough changes in the system, the modal amplitudes $\left\{C_{j}\right\}$ can be considered as effectively constant. We exploit this convenient aspect of this formulation when exploring the consequences of secular resonant sweeping during planetary migration.

\subsection{Linear Solution of the Present-day Solar System}

We have calculated the normal mode solution for the eccentricity evolution of a six-planet system consisting of Jupiter and Saturn and the terrestrial planets. Uranus and Neptune have been omitted from this treatment for simplicity as their influence on Jupiter and Saturn is weak and not efficiently communicated to the inner solar system.

The system's orthonormal eigenvectors $\left(\mathbf{v}_{j}\right)$ are shown in Figure 1 with their associated eigenfrequencies $\left(g_{j}\right)$. As is the convention for the solar system, the eigenmode index $(j)$ identifies the 
planet that has the largest component in the eigenvector (i.e., Mercury has the largest component in the $j=1$ mode and its evolution is strongly governed by this mode, Venus has the largest component in the $j=2$ mode, etc.). Large components for multiple planets in a single eigenvector indicate strong coupling between those planets. The sign of an eigenvector component $\left(v_{i j}\right)$ indicates the relative apsidal orientation within the mode, with like signs indicating alignment and opposite signs anti-alignment.

The eigenvectors of this six-planet system can be divided into two groups, those where the terrestrial planets have the largest components $(j=1-4)$ and those where Jupiter and Saturn have the dominant components $(j=5-6)$. This trend in the structure of the eigenvectors is indicative of the weak coupling between the terrestrial and giant planets in their current configuration. The eigenvector signatures of the terrestrial modes $(j=1-4)$ each have large components for at least two planets indicating significant coupling in their evolution.
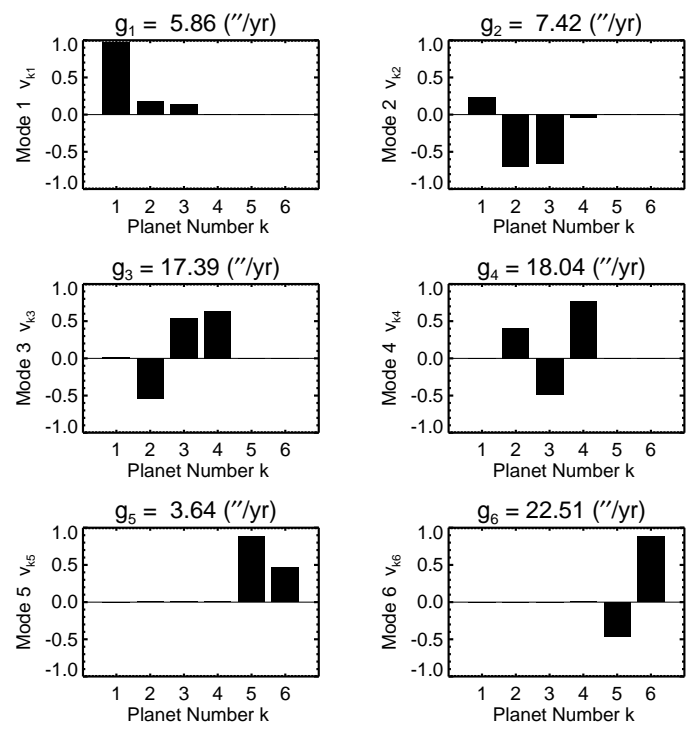

Fig. 1.- Orthonormal eigenvectors of our six-planet model including Mercury through Saturn. Each eigenvector is labeled with its eigenfrequency. Large components for multiple planets indicate strong coupling between those planets in the mode. The corrections due to nearby first-order mean motion resonances were included.

Using the VSOP82 ephemeris of Bretagnon (1982), we have computed the initial amplitudes and phases of this system's normal modes. The fraction of the system's AMD present in each mode $\left(C_{j}^{2} / 2\right)$ is shown in Figure 2, The modal eccentricity amplitudes are listed in Table1, The $j=5,6$ modes of Jupiter and Saturn contain the majority of the system's AMD 1 Among the modes that

\footnotetext{
${ }^{1}$ The neglected contributions of Uranus and Neptune (and the resulting $j=7,8$ modes) amount to an additional $2 \%$ of the system AMD.
} 
control the evolution of the terrestrial planets the $j=1$ and $j=4$ mode amplitudes contain $\sim 85 \%$ of the terrestrial subsystem's AMD (i.e., the sum $\Sigma_{j=1}^{4} C_{j}^{2} / 2$ ). The $j=1$ and $j=4$ modes strongly affect the evolution of Mars and Mercury and are responsible for the large eccentricities of these planets. Similarly, the $j=2,3$ modes strongly affect the evolution of Venus and Earth. The relatively small amplitudes of these modes are manifested as smaller eccentricities of Venus and Earth.

This dynamical configuration and partitioning of the AMD between the terrestrial planets is peculiar. The terrestrial eigenfrequencies with the largest amplitudes are both in close proximity to an eigenfrequency with a much smaller amplitude. For example, the $j=1$ and $j=2$ frequencies are close with $g_{1}=5.86^{\prime \prime} \mathrm{yr}^{-1}$ and $g_{2}=7.42^{\prime \prime} \mathrm{yr}^{-1}$, and the amplitude ratio is $C_{1} / C_{2} \approx 5$. Similarly, the $j=3$ and $j=4$ modes that strongly affect Earth and Mars are close in value with $g_{3}=17.39^{\prime \prime} \mathrm{yr}^{-1}$ and $g_{4}=18.04^{\prime \prime} \mathrm{yr}^{-1}$ and have an amplitude ratio of $C_{4} / C_{3} \approx 2$.

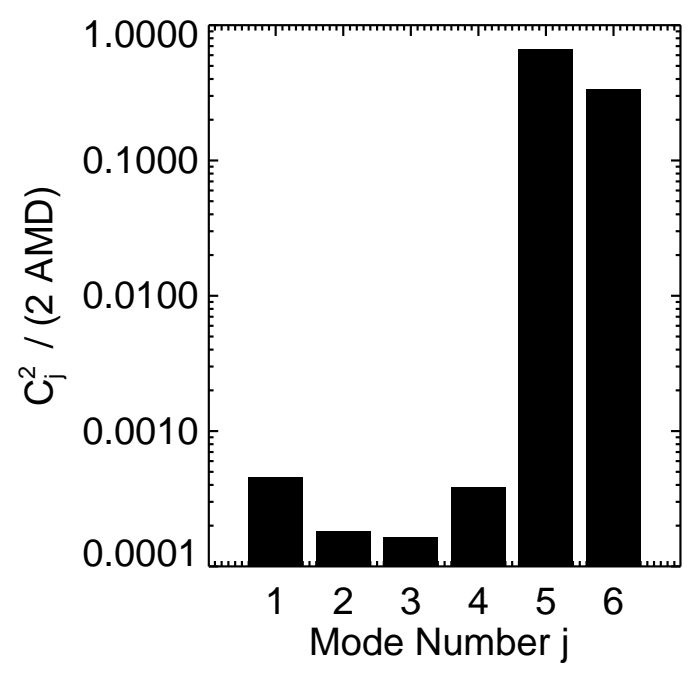

Fig. 2.- Fraction of the solar system's angular momentum deficit (AMD) in each eccentricity mode is shown. Note: $\mathrm{AMD}=\sum_{j} C_{j}^{2} / 2$.

The frequencies of the terrestrial modes (i.e., $j=1-4$ ) all take values between those that dominate the present-day evolution of Jupiter and Saturn (i.e., $g_{5}<g_{1-4}<g_{6}$ ). As a result the giant planet precession frequencies need not be grossly modified to resonate with terrestrial modes (e.g., $g_{5}=g_{1}$ ). Meteorites and asteroids placed in or near a secular resonance with the giant planets may be strongly perturbed to eccentric orbits (Farinella et al. 1993), and may even be driven into the Sun (e.g., Gladman et al. 1997; Chambers \& Wetherill 1998).

Linear secular theory has a number of properties that make it a useful tool for exploring the implications of giant planet migration. As described above, the secular frequencies $\left\{g_{j}\right\}$ and 


\begin{tabular}{|rc|rrrrrr|}
\hline & & \multicolumn{6}{|c|}{ Mode Index $j$} \\
Planet & $i$ & 1 & \multicolumn{1}{|c|}{2} & \multicolumn{1}{c|}{3} & \multicolumn{1}{c|}{4} & \multicolumn{1}{c|}{5} & \multicolumn{1}{c|}{6} \\
\hline Mercury & 1 & 0.1808 & 0.0216 & 0.0164 & 0.0015 & -0.0373 & -0.0162 \\
Venus & 2 & 0.0097 & -0.0185 & -0.0166 & -0.0017 & 0.0191 & 0.0094 \\
Earth & 3 & 0.0005 & -0.0119 & 0.0111 & 0.0202 & -0.0017 & -0.0066 \\
Mars & 4 & 0.0010 & -0.0248 & 0.0277 & -0.0673 & 0.0012 & 0.0100 \\
Jupiter & 5 & 0.0000 & 0.0000 & 0.0000 & 0.0000 & 0.0433 & 0.0162 \\
Saturn & 6 & 0.0000 & -0.0000 & 0.0000 & 0.0000 & -0.0357 & 0.0484 \\
\hline
\end{tabular}

Table 1: Eccentricity Amplitudes $\left(e_{i j}\right)$ Computed in Our Six-planet Secular Model Using the Ephemeris of Bretagnon (1982)

eigenvectors $\left\{\mathbf{v}_{j}\right\}$ are functions of the planet masses $\left\{m_{i}\right\}$ and semimajor axes $\left\{a_{i}\right\}$. This allows us to compute the evolution of the system's eigenfrequencies, and their sweeping through the solar system, for various orbital migration histories of the planets. When a system's mass distribution does not change, its mode amplitudes $\left\{C_{j}\right\}$ are integrals of the system's time evolution. For the solar system, the planetary precession periods are $\sim 10^{4}-10^{5} \mathrm{yr}$. As long as the mass distribution of the system evolves on longer timescales and the system is not near resonance, the modal amplitudes $\left\{C_{j}\right\}$ may be considered effectively constant (Ward 1981).

\subsection{Secular Precession Near First-order Mean Motion Resonances}

In the standard Laplace-Lagrange model the direct terms of the disturbing function containing the mean longitudes and all the indirect terms in the disturbing function may be neglected when averaging over long timescales. However, near commensurabilities between the mean motions of planets, resonant terms in the disturbing function can make significant contributions to orbit precession. A well-known example of this in the solar system is the so-called Great Inequality which results from the close proximity of Jupiter and Saturn to their mutual 5:2 MMR. Jupiter and Saturn's nearness to this resonance modifies the $g_{5}$ and $g_{6}$ frequencies by $\approx+0.5^{\prime \prime} \mathrm{yr}^{-1}$ and $+6^{\prime \prime} \mathrm{yr}^{-1}$ respectively (see, e.g., Brouwer \& Clemence 1961; Murray \& Dermott 1999). The lowestorder mean motion resonances encountered during the divergent migration of Jupiter and Saturn are the 2:1 and 3:2. Both are first-order resonances and may strongly affect the secular evolution of the planets.

Malhotra et al. (1989) developed an approach for including the influence of first-order mean motion resonances in the secular model and used it to account for the evolution of the Uranian satellites. Their treatment of the problem includes the averaged influence of the direct terms from the disturbing function related to first-order $(\ell: \ell-1)$ mean motion resonances. The $2: 1$ is unusual among the first-order resonances as it also gives rise to indirect terms in the disturbing function of 
both planets (cf. Murray \& Dermott 1999).
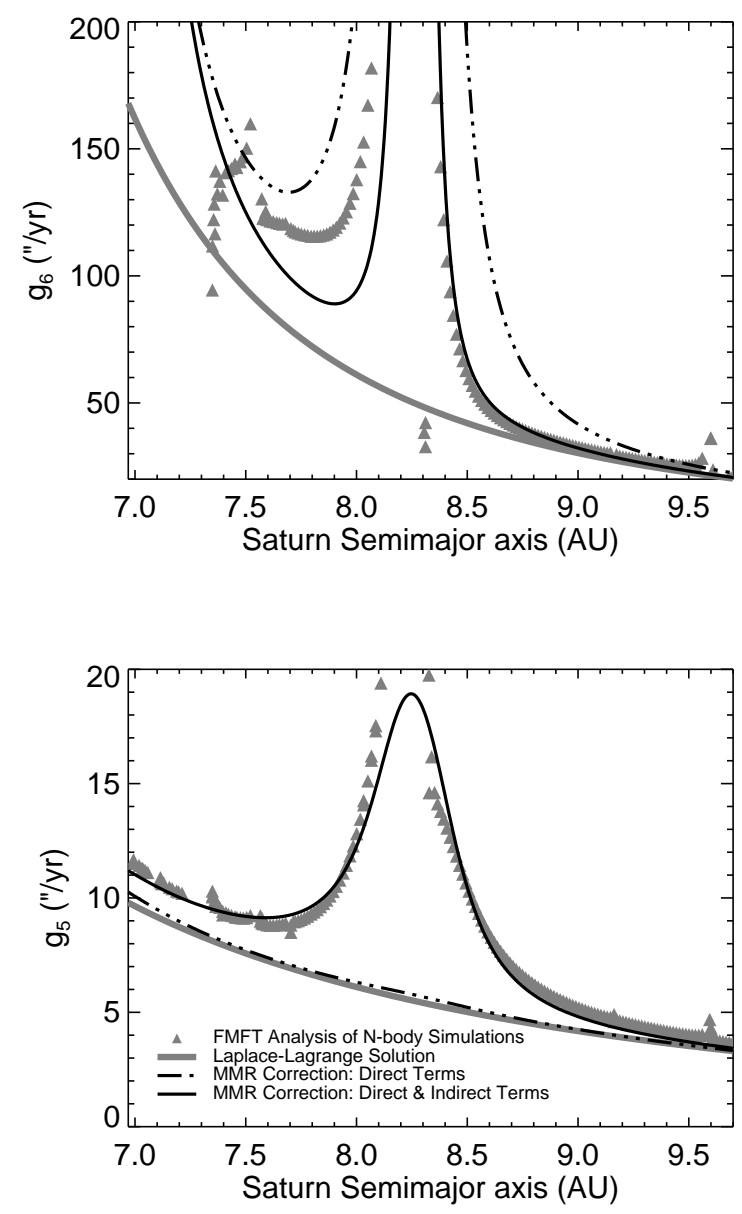

Fig. 3.- Comparing results of modified secular theory and $N$-body simulations. Jupiter orbits with a semimajor axis of 5.2 AU. Saturn's semimajor axis is changed from 7 to 9.7 AU. The apsidal eigenfrequencies of the $g_{6}$ and $g_{5}$ modes were computed using standard Laplace-Lagrange theory (gray solid line), with the direct terms of from the disturbing function of first-order mean motion resonances (dash-dotted black line) and with both direct and indirect contributions from firstorder mean motion resonances (black solid lines). Also shown are results from individual $N$-body integrations that have been Fourier analyzed to identify the characteristic precession frequencies of Jupiter and Saturn (gray triangles).

Figure 3 shows the apsidal eigenfrequencies of Jupiter and Saturn as a function of Saturn's semimajor axis determined using several different methods. In each case, Jupiter's semimajor axis was held constant at 5.2 AU, the semimajor axis of Saturn was increased from just outside the mutual 3:2 mean motion resonance at about $6.8 \mathrm{AU}$, across the 2:1 at 8.25 AU, to $9.7 \mathrm{AU}$. 
To determine the eigenfrequencies of the Jupiter-Saturn system as a function of their semimajor axes we performed a set of individual $N$-body simulations, each with a different initial semimajor axis for Saturn. In each simulation the planets were assigned nearly circular, nearly coplanar orbits (i.e., $e \simeq 0.005, I=e / 2$ ), random phase elements and were evolved for about $11 \mathrm{Myr}$. The SyMBA integrator (Duncan et al. 1998) was used in these simulations and the precessional effects of general relativity were included (e.g., Quinn et al. 1991).

We determined the eccentricity amplitudes $\left(e_{i j}\right)$ and eigenfrequencies of the system by analyzing the evolution of a planet's eccentricity vector $\left(h_{i}, k_{i}\right)$ using a Frequency Modified Fourier Transform code (hereafter FMFT; Sidlichovsky \& Nesvorny 1997) 2 For each simulation the two frequencies with the largest amplitudes in the evolution of Jupiter's eccentricity vector $\left(h_{5}, k_{5}\right)$ are shown in Figure 3 with gray triangles. The close proximity of the measured frequencies to the eigenfrequencies of the Jupiter-Saturn system identifies them as the $g_{5}$ and $g_{6}$. Since the $N$-body simulations include all gravitational interactions between the planets, resonant, and otherwise, these FMFT results serve as "measurements" of the apsidal eigenfrequencies of the actual system and can be used to assess the fidelity of more approximate, but analytically tractable, treatments.

As Saturn's orbit is expanded, the values of the $g_{5}$ and $g_{6}$ frequencies show two important characteristics. First, near both the 3:2 and 2:1 MMRs the value of the $g_{6}$ frequency diverges. The behavior of the $g_{5}$ is somewhat different, showing divergence behavior only near the 2:1 mean motion resonance. For orbital separations outside the 2:1 resonance (i.e., the semimajor axis of Saturn greater than 8.25AU in this case), the two eigenfrequencies decrease as Jupiter and Saturn continue to diverge.

We have used secular theory to compute the modal frequencies in three different ways. The standard Laplace-Lagrange treatment is shown as a gray solid line in both figures. This treatment contains no information regarding mean longitudes of the planets and fails to account for divergence in the $g_{5,6}$ frequencies near the 3:2 and 2:1 mean motion resonances.

We also computed the apsidal eigenfrequencies of Jupiter and Saturn, correcting the LaplaceLagrange treatment with the direct terms of Malhotra et al. (1989). The value of $g_{5}$ and $g_{6}$ are shown with a dash-dotted line. The corrections due to the direct terms account for divergence of the $g_{6}$ frequency when approaching the $3: 2$ or 2:1 resonances, but fail to account for the changes in the $g_{5}$ frequency due to the $2: 1$ resonance.

Finally, we compute the apsidal eigenfrequencies using the correction of both direct and indirect terms from the first-order mean motion resonances. The contributions of the indirect terms associated with the 2:1 MMR may be included in the model of Malhotra et al. (1989) by adding $-2 \alpha \delta_{j, 1}$ to their Equation (26b) where $\delta_{j 1}$ is the kronecker delta function (R. Malhotra, private communication 2011) 3 As the 2:1 is the only first-order resonance with indirect terms it is also

\footnotetext{
${ }^{2}$ The FMFT code used in this study was derived from that used in Christou \& Murrav (1999).

${ }^{3} \mathrm{~A}$ more cumbersome inclusion of the indirect terms that yields similar results may also be derived from the
} 
the only resonance affected by this amended theory.

The inclusion of the indirect terms dramatically improves the agreement between the secular frequencies computed with FMFT-measured values and the corrected secular theory and accounts for the behavior of the $g_{5}$ frequency around the 2:1 MMR. This model assumes that the resonant argument is rapidly circulating and consequently becomes less accurate near the resonant separatrix where the circulation rate slows. The continuous behavior of the corrected $g_{5}$ frequency as the $2: 1$ MMR is crossed, its inflection and local maxima are model artifacts of these assumptions. For Jupiter and Saturn more than $\sim 0.1$ AU outside the 2:1 MMR resonance, the results of the $N$-body simulations and the modified secular theory are in close agreement.

The influence of additional mean motion resonances between Jupiter and Saturn are also visible in FMFT measurements of $g_{5}$ and $g_{6}$. These appear as deviation from the smooth general trends indicated by secular theory. The effects of the 5:3 and 7:4 MMRs when Saturn is near 7.3 and 7.55 AU and the 5:2 MMR as Saturn approaches its current orbit at 9.54 AU are evident. These secondand third-order resonances are relatively weak compared to the 3:2 and 2:1 and their influence on the eigenfrequencies is both smaller and localized to configurations close to exact resonance.

These results clearly demonstrate that the divergent migration of Jupiter and Saturn across their 2:1 MMR, as suggested by Tsiganis et al. (2005) and Gomes et al. (2005), results in secular resonant sweeping of the $g_{5}$ across the values of all four of the current terrestrial eigenfrequencies. Thus, this migration scenario may subject the terrestrial planets to potentially destabilizing secular resonant perturbations.

\section{Excitation Due to Secular Resonant Crossing}

Excitation via passage through linear secular resonances with the giant planets has been considered previously in several contexts (Ward et al. 1976; Heppenheimer 1980; Nagasawa et al. 2005). First, we describe the test particle solutions of Ward et al. (1976) and Nagasawa et al. (2005) adapting them to the case of resonant sweeping due to planetary migration 4 We then extend this model to describe resonances with the eigenmodes of the terrestrial planets using the subsystem approximation of Ward (1981).

disturbing function for osculating elements (see, e.g., Ellis \& Murray 2000; Murray \& Dermott 1999).

${ }^{4}$ See also Gomes (1997) and the recent work of Minton \& Malhotra (2011) that have used similar models to evaluate the migration induced sweeping of the $g_{6}$ and $s_{6}$ resonances through the asteroid belt. 


\subsection{Test Particle Excitation}

Consider a test particle in a system where planetary migration causes its orbital precession frequency $(g)$ to pass through a resonance with one of the system's eigenfrequencies $\left(g_{j}\right)$. In a reference frame that rotates with frequency $g_{j}$, the equations of motion of $h=e \sin \left(\varpi-g_{j} t-\beta_{j}\right)$ and $k=e \cos \left(\varpi-g_{j} t-\beta_{j}\right)$ for the planetesimal interacting with the resonant mode are

$$
\begin{aligned}
& \frac{d h}{d t}-\left(g-g_{j}\right) k=\mu_{j} \\
& \frac{d k}{d t}+\left(g-g_{j}\right) h=0,
\end{aligned}
$$

where

$$
\mu_{j}=\frac{n a}{4} \sum_{i} e_{i j}\left(\frac{m_{i}}{M_{\odot}}\right) \frac{\alpha}{a_{>}} b_{3 / 2}^{2}
$$

An analytic solution for resonance passage can be obtained by introducing a new independent variable $\phi \equiv \int \Delta g d t$, where $\Delta g=g-g_{j}$ and transforming Equations 13 and 14 into

$$
\begin{gathered}
\frac{d h}{d \phi}-k=\frac{\mu_{j}}{\Delta g} \\
h+\frac{d k}{d \phi}=0 .
\end{gathered}
$$

The particular solution of this expression is

$$
\begin{gathered}
h=\left(C_{1}+c\right) \cos \phi+\left(S_{1}+s\right) \sin \phi \\
k=-\left(C_{1}+c\right) \sin \phi+\left(S_{1}+s\right) \cos \phi
\end{gathered}
$$

with

$$
\begin{aligned}
C_{1} & =\int\left(\frac{\mu_{j}}{\Delta g}\right) \cos \phi d \phi \\
S_{1} & =\int\left(\frac{\mu_{j}}{\Delta g}\right) \sin \phi d \phi .
\end{aligned}
$$

Defining $t=0$ as the time of exact resonance $\left(g=g_{j}\right)$ and setting $\Delta \dot{g}=\dot{g}-\dot{g}_{j}$ where $\dot{g}_{j}=\left|d g_{j} / d t\right|$, the variable $\phi=-t^{\prime 2}=-\frac{1}{2} \Delta \dot{g} t^{2}=-\frac{1}{2}(\Delta g)^{2} / \Delta \dot{g}$, the solutions for $h, k$ following resonance passage are

$$
\begin{gathered}
h_{\mathrm{res}}=-\mu_{j}\left(\frac{\pi}{\Delta \dot{g}}\right)^{1 / 2}\left[\left(C_{1}\left(t^{\prime}\right)+\frac{1}{2}\right) \cos t^{\prime 2}+\left(S_{1}\left(t^{\prime}\right)+\frac{1}{2}\right) \sin t^{\prime 2}\right] \\
k_{\mathrm{res}}=\mu_{j}\left(\frac{\pi}{\Delta \dot{g}}\right)^{1 / 2}\left[\left(S_{1}\left(t^{\prime}\right)+\frac{1}{2}\right) \cos t^{\prime 2}-\left(C_{1}\left(t^{\prime}\right)+\frac{1}{2}\right) \sin t^{\prime 2}\right]
\end{gathered}
$$

where $S_{1}$ and $C_{1}$ are the Fresnel sine and cosine integrals and we have required that $h, k \rightarrow 0$ as $t \rightarrow-\infty$. 
Long after resonance passage, as $t \rightarrow \infty$, the test particle's new free eccentricity is

$$
e_{\text {res }}=\mu_{j}\left(\frac{2 \pi}{|\Delta \dot{g}|}\right)^{1 / 2}
$$

In essence, resonance passage excites a new contribution to the free eccentricity of the body. The amplitude of this response is determined by the rate of passage through resonance and is a function of the migration rate. It is convenient to use an exponentially decaying migration rate of the form

$$
\frac{d a_{i}}{d t}=\frac{\Delta a_{i}}{\tau}
$$

where $\Delta a_{i}=a_{i, f}-a_{i}$ is the distance of the planet from its final semimajor axis $\left(a_{i, f}\right)$ and $\tau$ is the characteristic migration timescale. We treat $\tau$ as a global migration constant and use this to parameterize migration speed. We note that Equation (25) can also be used to convert between analytic constraints, that are easily expressed as an exponential timescale $(\tau)$, and migration rates $\left(d a_{i} / d t\right)$ which might be more easily measured from $N$-body simulations of planetary migration. Using the exponential migration model,

$$
\Delta \dot{g}=\sum_{i}\left(\frac{d g}{d a_{i}}-\frac{d g_{j}}{d a_{i}}\right) \frac{\Delta a_{i}}{\tau}
$$

where all terms except the timescale are functions of the planetary masses and semimajor axes at the time of resonance. This can also be used to identify a critical migration timescale $\left(\tau^{*}\right)$ required to excite the eccentricity to some prescribed value $e_{\text {res }}$

$$
\tau^{*}=\frac{1}{2 \pi}\left|\sum_{i}\left(\frac{d g}{d a_{i}}-\frac{d g_{j}}{d a_{i}}\right) \Delta a_{i}\right|_{r}\left(\frac{e_{\mathrm{res}}}{\mu_{j}}\right)^{2},
$$

where the mass and orbital state of the perturbing planets are encapsulated in $\mu_{j}$ (see Equation (15)). If the test particle's free eccentricity prior to resonance passage was zero, then Equation (24) is the new eccentricity after passage of the secular resonance. However, when the free eccentricity is not initially zero, the initial secular coordinates $h_{o}, k_{o}$ add vectorally with those obtained from resonance passage $h_{\text {res }}, k_{\text {res }}$. The two contributions may interfere constructively or destructively depending on their relative phase $\gamma$ (Ward et al. 1976; Minton \& Malhotra 2008) and the resulting eccentricity can be determined from geometric considerations

$$
e_{f}=\left(e_{o}^{2}+e_{\text {res }}^{2}+2 e_{o} e_{\text {res }} \cos \gamma\right)^{1 / 2}
$$

(Heppenheimer 1980). Recognizing the phase between contributions as effectively random and isotropic allows Equation (28) to be used to as a distribution function of resulting eccentricities. For example, given amplitudes for the initial and resonant eccentricity vectors, $\gamma=0, \pi / 2$, and $\pi$ yield the maximum, median, and minimum eccentricities expected. 


\subsection{Subsystem Approximation for Terrestrial-Giant Planet Interactions}

Due to their much smaller mass and modal amplitudes, the terrestrial planets have a negligible influence on the evolution of the giant planets. The subsystem approximation of Ward (1981) treats the terrestrial planets and giant planets as separate and independently evolving secular systems

and then examines their interaction. A brief summary of this model is described below (see Ward 1981, for the complete development).

In this treatment the secular evolution matrix $\mathbf{A}$, whose elements are defined in Equations (37) and (4), is divided into submatricies that describe coupling within each terrestrial and giant planet subgroup and between the subgroups

$$
\mathbf{A}=\left(\begin{array}{cc}
\mathbf{A}_{T} & \mathbf{P} \\
\tilde{\mathbf{P}} & \mathbf{A}_{G}
\end{array}\right)
$$

Here $\mathbf{A}_{T}$ is the terrestrial $4 \times 4$ submatrix and $\mathbf{A}_{G}$ is the $2 \times 2$ submatrix describing Jupiter and Saturn. The direct influence of the giant planets on the terrestrial planets' precession frequencies is included through the diagonal elements of $\mathbf{A}_{T}$ (see Equation (3)). The coupling between the terrestrial and giant planet modes are included through the $4 \times 2$ and $2 \times 4$ off-diagonal submatrices of $\mathbf{A}$ and are labeled as $\tilde{\mathbf{P}}$ and $\mathbf{P}$.

The solutions for each subgroup are assumed to be independent. As before we label the solution to the $4 \times 4$ terrestrial subgroup $\left(\mathbf{A}_{T}\right)$

$$
\left\{\begin{array}{l}
H \\
K
\end{array}\right\}=C_{j} \mathbf{v}_{j}\left\{\begin{array}{c}
\sin \left(g_{j} t+\beta_{j}\right) \\
\cos \left(g_{j} t+\beta_{j}\right)
\end{array}\right\}
$$

with $j=1-4$. Similarly, the solution for the Jupiter-Saturn subgroup $\left(\mathbf{A}_{G}\right)$ is

$$
\left\{\begin{array}{l}
H \\
K
\end{array}\right\}=D_{n} \mathbf{u}_{n}\left\{\begin{array}{c}
\sin \left(\nu_{n} t+\delta_{n}\right) \\
\cos \left(\nu_{n} t+\delta_{n}\right)
\end{array}\right\},
$$

where $\nu_{n}$ and $\mathbf{u}_{n}$ are the two-planet eigenfrequencies and eigenvectors, $D_{n}$ and $\delta_{n}$ are the amplitude and phase of the two-planet modes, and we retain the index labels $n=5,6$ conventionally used for these modes in the solar system. Because the terrestrial planets only weakly affect the precession of the giant planets, the eigenmodes of the two- and four-planet subgroups are nearly identical to those of the six-planet system (i.e., $\nu_{n}=g_{n}$, for $n=5,6$ ) shown in Figures 1 and 2. Hereafter we refer to the giant planet eigenfrequencies with the symbol $\nu_{n}$.

\subsection{The Sweeping $\nu_{5}$ Resonance during Migration}

Figure 4 shows the four eigenfrequencies of the terrestrial subgroup along with the $\nu_{5}$ frequency as a function of Saturn's semimajor axis. As before, Jupiter is held fixed near its current orbit at 


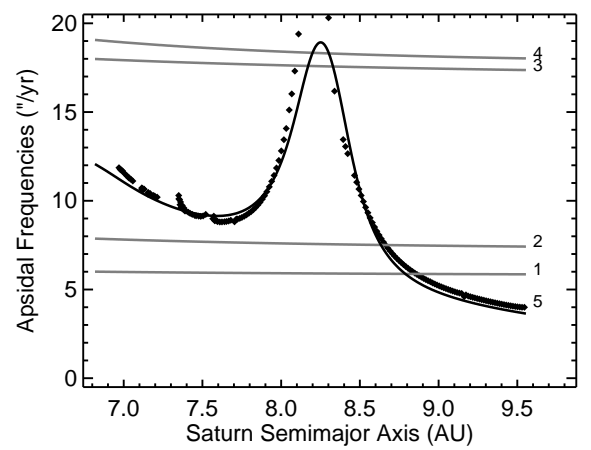

Fig. 4.- Eigenfrequencies computed using the subgroup approximation as a function of Saturn's semimajor axis. Each frequency is labeled with its mode index $j$ at the right. Jupiter is located at $5.2 \mathrm{AU}$. The $j=1-4$ modes that describe the terrestrial planets are shown with gray lines. The $\nu_{5}$ frequency computed with modified secular theory secular is shown with a black solid line. Black diamonds show the FMFT measurements of the $\nu_{5}$ from a series of $N$-body simulations. The $\nu_{5}$ mode, associated with Jupiter, resonates with each of the terrestrial modes during the divergent migration of Jupiter and Saturn.

5.2 AU. The terrestrial frequencies are predominantly determined by their mutual interactions and Jupiter's proximity and are only weakly influenced by Saturn's orbital position. This is illustrated in Figure 4 as the terrestrial frequencies ( $g_{1-4}$ shown in gray) are nearly constant as Saturn's orbital radius expands.

The $\nu_{5}$ frequency decreases from about $12^{\prime \prime} \mathrm{yr}^{-1}$ to about $8.5^{\prime \prime} \mathrm{yr}^{-1}$ as Saturn's orbit expands from just outside the 3:2 mean motion resonance with Jupiter to about 7.8 AU. The small gaps in the FMFT measurement for the $\nu_{5}$ frequency when Saturn is at 7.3 AU and 7.55 AU result from the dispersion of forcing frequencies introduced by the nearby 5:3 and 7:4 MMRs between Jupiter and Saturn. As Saturn's orbit expands beyond 7.8 AU toward the 2:1 with Jupiter near 8.25 AU, the influence of the 2:1 MMR dominates over the linear secular terms and the $\nu_{5}$ frequency increases from $8.5^{\prime \prime} \mathrm{yr}^{-1} \rightarrow 20^{\prime \prime} \mathrm{yr}^{-1}$. It resonates with the $g_{3}$ and $g_{4}$ terrestrial frequencies when Saturn is near 8.17 AU and 8.20 AU, respectively.

As Saturn's orbital radius is increased outside the 2:1, the effect of the mean motion resonance weakens with distance and the $\nu_{5}$ frequency again decreases from about $20^{\prime \prime} \mathrm{yr}^{-1}$ to $4^{\prime \prime} \mathrm{yr}^{-1}$ as Saturn's orbit expands from 8.3 AU to 9.54 AU. The $\nu_{5}$ resonates a second time with both the $g_{4}$ and $g_{3}$ just exterior to the 2:1 MMR when Saturn is near 8.30 AU and 8.33 AU. As Saturn's orbit is expanded further, the $\nu_{5}$ resonates with the $g_{2}$ and $g_{1}$ when Saturn is near $8.63 \mathrm{AU}$ and 8.79 AU. In total we have identified six individual secular resonances between the $\nu_{5}$ frequency and a terrestrial eigenmode that occurs as a result of the divergent migration, of Jupiter and Saturn, from just outside their mutual 3:2 mean motion resonance to their present orbits. The semimajor 
axes of Saturn for each of these resonances is listed in Table 2, In their numerical exploration of this problem, Brasser et al. (2009) parameterized the giant planet configuration at the time of resonance with the ratio of the orbital periods of Saturn to Jupiter $\left(P_{S} / P_{J}\right)$. Because the value of the period ratio $\left(P_{S} / P_{J}\right)$ at $g_{j}-\nu_{5}$ resonances is weakly dependent on Jupiter's semimajor axis this ratio can be used as a convenient independent variable to approximately identify resonant configurations. Its value for each secular resonance is listed in Table 2.

\subsection{Secular Resonances, "Speed Traps" and Critical Migration Parameters}

Using the subgroup approximation, Ward (1981) showed that tuning the system through a linear secular resonance between the terrestrial and giant planets (i.e., $\nu_{n}=g_{j}$ ) excites the terrestrial mode amplitude by an amount

$$
C_{j, \text { res }}=D_{n} F_{j n}\left(\frac{2 \pi}{\left|\Delta \dot{g}_{j n}\right|}\right)^{1 / 2}
$$

where $\Delta \dot{g}_{j n}=\dot{g}_{j}-\dot{\nu}_{n}$. Here $F_{j n}$ is an effective width of the resonance given by

$$
F_{j n}=\tilde{\mathbf{v}}_{j} \cdot \mathbf{P} \cdot \mathbf{u}_{n}
$$

where $\tilde{\mathbf{v}}_{j}$ is the transpose of $\mathbf{v}_{j}$. Equation (32) is the generalization of the test particle response of resonance passage (Equation (24) ) to a four-planet terrestrial subsystem. Here the mode amplitudes $C_{j}, D_{n}$ take the place of modal eccentricities, and the functional dependence on the migration rate through $\Delta \dot{g}_{j n}$ is identical to the test particle case.

The AMD exchanged with the terrestrial planets through the resonance is

$$
C_{j, \mathrm{res}}^{2}=D_{n}^{2} \tau\left[2 \pi F_{j n}\left|\sum_{i}\left(\frac{d g_{j}}{d a_{i}}-\frac{d \nu_{n}}{d a_{i}}\right) \Delta a_{i}\right|_{r}^{-1}\right] .
$$

The excitation of the terrestrial mode depends on three principal factors: the configuration of the planetary system at resonance (determined by the value of the terms in square brackets), the amplitude of the resonant giant planet mode $\left(D_{5}\right)$, and the migration timescale $(\tau)$.

It is convenient to combine the forcing amplitude and migration timescale into a single migration parameter

$$
\hat{\tau}=\left(\frac{D_{5}}{D_{5, \mathrm{obs}}}\right)^{2} \tau=\left(\frac{e_{55}}{0.0433}\right)^{2} \tau .
$$

The value of the migration parameter is proportional to the AMD exchange and can be used to identify the family of giant planet eccentricity states (e.g., $\left.e_{55}\right)$ and migration timescales $(\tau)$ that produce a certain level of excitation. In this definition, we have scaled the migration parameter so that $\hat{\tau}=\tau$ when Jupiter and Saturn migrate with eccentricities equal to their present-day values. 
Using the above relations we identify the critical migration parameter for each resonance $\left(\hat{\tau}_{j n}^{*}\right)$ capable of exciting a terrestrial mode to its observed amplitude

$$
\hat{\tau}_{j n}^{*}=\frac{1}{2 \pi F_{j n}^{2}}\left(\frac{C_{j, \mathrm{obs}}}{D_{n, \mathrm{obs}}}\right)^{2}\left|\sum_{i}\left(\frac{d g_{j}}{d a_{i}}-\frac{d \nu_{n}}{d a_{i}}\right) \Delta a_{i}\right|_{r} .
$$

In a sense, passage through the secular resonance may be used as a dynamical "speed trap" to constrain the eccentricity state and maximum migration timescale (or minimum migration rates) of Jupiter and Saturn at the time of resonance. For each secular resonance we have computed the giant planet orbits, resonance widths $\left(F_{j n}\right)$, and critical migrations parameters $\left(\hat{\tau}_{j n}^{*}\right)$ for exciting the resonant terrestrial mode to its observed amplitude. The characteristics describing these resonances are listed in Table 2, Equation (32) indicates that resonant excitation is dependent on the slope $d \nu_{5} / d a_{6}$. The $g_{3}-\nu_{5}$ and $g_{4}-\nu_{5}$ secular resonances occur when Jupiter and Saturn are very near the 2:1 MMR and the modified secular model becomes less accurate. For these, we use values for the $\nu_{5}$ frequency gradient interpolated from the FMFT results in Figure 4 .

The effective width of a resonance $\left(F_{j n}\right)$ is determined by the projection of the resonant terrestrial eigenvector $\left(\mathbf{v}_{j}\right)$ onto the off-diagonal matrix $\mathbf{P}$ that accounts for coupling between the terrestrial and giant planet subgroups. As these terms scale with planet mass (see, e.g., Equation (41)), the effective width tends to be larger for resonances with modes where more massive planets have larger eigenvector components $\left(v_{k j}\right)$. The resonance widths are shown in Table 2. Since the $j=2,3$ modes have the largest eigenvector components for Earth and Venus (see Figure 11) resonances with these modes have greater effective widths than resonances with the $j=1,4$ modes.

Also, the greater effective widths of the resonances with the $j=2,3$ modes and Equation (32) indicate that for a given frequency sweeping rate at resonance $\left(\Delta \dot{g}_{j n}\right)$, the $j=2,3$ modes experience a greater excitation of their amplitudes than the $j=1,4$ modes. This susceptibility to excitation appears in contrast to the mode amplitude partitioning of the terrestrial planets, where $C_{2}$ and $C_{3}$ are smaller than $C_{1}$ and $C_{4}$ (see Figure 2).

This general model can be used to predict the excitation of modal eccentricity amplitudes due to passage through a secular resonance $\left(e_{i j, \text { res }}\right)$

$$
e_{i j, \mathrm{res}}=e_{i j, \mathrm{obs}}\left(\frac{\hat{\tau}}{\hat{\tau}_{j 5}^{*}}\right)^{1 / 2}
$$

where $e_{i j, \mathrm{obs}}$ are the eccentricity amplitudes of the present-day system listed in Table 1 Note that in both the test particle and subgroup approximation the resonant forcing of eccentricity scales as $\hat{\tau}^{1 / 2}$ (or equivalently $e_{\text {res }} \propto e_{55} \tau^{1 / 2}$ ).

These relations and the observed state of the terrestrial planets can be used to constrain the giant planet migration parameter at the time of passage through a secular resonance. For example, if we know Jupiter's eccentricity at the time of resonance (e.g., the current value) then we can constrain the migration timescale of Jupiter and Saturn (and vice versa). 


\begin{tabular}{ccccccc}
\hline \multicolumn{2}{c}{ Mode } & Saturn & Period Ratio & Frequency \\
$j$ & $n$ & $a_{S}(\mathrm{AU})$ & $P_{S} / P_{J}$ & $\begin{array}{c}\text { Resonance Width } \\
g_{j}\left({ }^{\prime \prime} \mathrm{yr}^{-1}\right)\end{array}$ & $\begin{array}{c}\text { Critical Migration Parameter } \\
F_{j n}\left({ }^{\prime} \mathrm{yr}^{-1}\right)\end{array}$ \\
\hline 3 & 5 & 8.17 & 1.968 & 17.59 & 0.040 & 0.598 \\
4 & 5 & 8.20 & 1.979 & 18.34 & -0.028 & 3.609 \\
4 & 5 & 8.30 & 2.016 & 18.30 & -0.025 & 1.840 \\
3 & 5 & 8.33 & 2.025 & 17.57 & 0.036 & 0.329 \\
2 & 5 & 8.63 & 2.138 & 7.51 & -0.052 & 0.050 \\
1 & 5 & 8.79 & 2.196 & 5.87 & 0.013 & 0.683 \\
\hline
\end{tabular}

Table 2: Secular Resonances During the Divergent Migration of Jupiter and Saturn (See e.g., Figure 4)

Note. In this model Jupiter's semimajor axis is constant at 5.2 AU.

The critical migration parameters $\left(\hat{\tau}_{j n}^{*}\right)$ required to excite the terrestrial modes to their observed amplitudes are listed in Table 2. All of these migration parameters are shorter than the migration timescales observed in self-consistent models of planetesimal-driven giant planet migration (e.g., $\tau \sim 5-20 \mathrm{Myr}$; Hahn \& Malhotra 1999; Gomes et al. 2004) by a factor ranging from a few to 200. These secular resonances constrain migration to occur with a smaller $e_{55}$ Jovian eccentricity amplitude and/or short migration timescales to avoid driving the terrestrial eccentricities to large values. Further, we note that resonances between the $\nu_{5}$ frequency and the $g_{2}$ and $g_{3}$ terrestrial modes, predominantly responsible for the small eccentricities of Earth and Venus, place the strongest constraints on the migration of Jupiter and Saturn. We compare these model predictions with the results of orbital integrations below.

\section{Jupiter and Saturn's Migration Near the 2:1 MMR}

As Jupiter and Saturn diverge across their mutual 2:1 MMR they perturb the terrestrial planets via at least five distinct dynamical events. The $g_{3}-\nu_{5}$ and the $g_{4}-\nu_{5}$ resonances are each encountered on both sides of the 2:1 MMR. In addition, the 2:1 MMR excites the eccentricity of Jupiter and Saturn on a timescale comparable to the circulation period of the resonant argument. Near the 2:1 MMR this is $\mathcal{O}\left(10^{3}\right)$ yr. Because the typical secular timescales of the terrestrial planets are more than an order of magnitude longer $\left(\gtrsim \mathcal{O}\left(10^{4}\right)\right)$, exciting the eccentricities of Jupiter and Saturn in this way can be thought of as rapid, non-adiabatic changes in the $C_{5}$ and $C_{6}$ secular modal amplitudes.

To clarify the implications of individual events our analysis separates the $g_{3}-\nu_{5}$ and $g_{4}-\nu_{5}$ secular resonances, that occur as Jupiter and Saturn migrate near the 2:1, from the $g_{2,1}-\nu_{5}$ resonances, that occur for larger Jupiter-Saturn separations. We analyze the dynamics of these individual resonances using both $N$-body simulations and secular theory and examine how they 
act in concert.

\subsection{Initial Conditions}

To clearly illustrate the role of these events using orbital integrations it is useful to start with terrestrial mode amplitudes $\left(C_{1-4}\right)$ that are initially very small. The initial conditions for these simulations were prepared in the following way. Jupiter was placed on its current orbit and Saturn at 7.2 AU. Both giant planets were given initial eccentricities of 0.01 and comparable inclinations. The terrestrial planets were given their current semimajor axes, small eccentricities, and inclinations $(e=0.01)$ and randomly chosen angular phases. This configuration was processed further by integrating it forward in time while applying an additional force to damp the eccentricities and inclinations of the terrestrial planets with a decay timescale of three million years. The timescale was chosen to be slow compared to the secular timescale so that it effectively damped the terrestrial mode amplitudes rather than the elements of a single planet (Agnor \& Ward 2002). For these initial conditions the FMFT-measured eccentricity amplitudes are $e_{i j} \lesssim 0.005$ for $i, j=1-4$ (for the terrestrial planets) and $e_{55} \simeq 0.015$ for Jupiter, about one-third its observed value. This value of $e_{55}$ is comparable to that suggested in Tsiganis et al. (2005). For these initial conditions, the critical migration timescales needed to excite the terrestrial modes to their observed values are about a factor of nine longer than the migration parameter listed in Table 2 (see Equation (35)).

We have performed a series of $N$-body orbital integrations using this initial state. In these models Saturn is forced to migrate outward across the $2: 1$, halting its migration at $8.5 \mathrm{AU}$. The migration force applied produces exponential migration with an $e$-folding timescale $(\tau)$ similar to that of Malhotra (1993). The starting and ending points of Saturn's migration leave the $\nu_{5}$ frequency between the $g_{2}$ and $g_{3}$ frequencies and avoids depositing the system near a secular resonance (see, e.g., Figure 4). This allows us to isolate the effects of individual events during migration and use FMFT analysis of the final states to measure the ultimate eccentricity amplitudes that result.

In this set of simulations we varied the migration timescale from $\tau=10^{4} \rightarrow 4 \times 10^{7}$ years. The low end of this range is near the secular timescale and illustrates the response of the system to rapid, essentially non-adiabatic, evolution. The upper end encompasses migration timescales suggested

by $N$-body simulations of planetesimal-driven giant planet migration (e.g., Hahn \& Malhotra 1999; Gomes et al. 2004).

Figure 5 shows an example of the coupling between the gas giant and terrestrial planets as Jupiter and Saturn are forced to migrate across their mutual 2:1 mean motion resonance with an $e$-folding timescale of $\tau=10 \mathrm{Myr}$. The top frame shows the evolution of the semimajor axes, pericenters, and apocenters of Jupiter and Saturn. Their divergent crossing of the 2:1 MMR occurs near $t / \tau=0.425$ and the excitation of the giant planet eccentricities is readily apparent as the increased separation of the pericenter and apocenter curves from the semimajor axes. The lower panel shows these same orbital parameters for the terrestrial planets. Mars' eccentricity is excited 
to a value similar to the observed one. As Earth and Venus have significant components in both the $j=3$ and $j=4$ modes (see, e.g., Figure 1), their excitation increases eccentricities of these planets as well. This excitation is coincident with Jupiter and Saturn migrating across the 2:1 MMR.
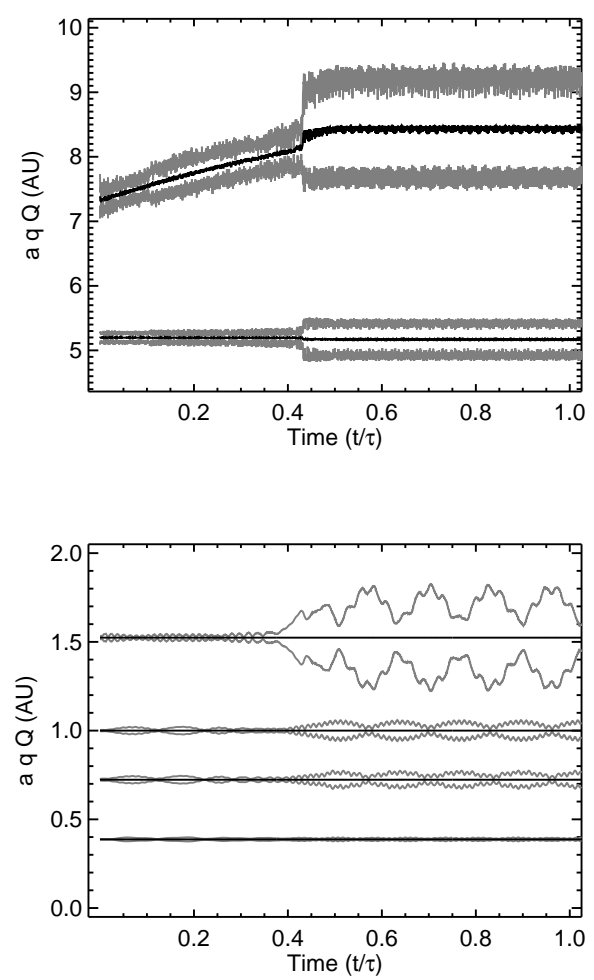

Fig. 5.- Forced migration simulation with $\tau=10 \mathrm{Myr}$. The semimajor axes of the Jupiter and Saturn and the terrestrial planets are shown with a black solid line. The pericenter and apocenter are shown in gray. The excitation of the terrestrial $j=3,4$ modes is evident in the increased eccentricity of Earth, Venus, and Mars.

\subsection{The First Crossing of the $g_{3}-\nu_{5}$ and $g_{4}-\nu_{5}$ Resonances}

Figure 6 shows the eccentricity excitation due to the first passage through the $g_{3}-\nu_{5}$ and $g_{4}-\nu_{5}$ secular resonances as a function of the migration timescale $\tau$. The theoretical predictions for the $j=3$ and $j=4$ eccentricity amplitudes of Earth and Mars computed with Equation (37) are shown with dotted and dashed lines. The maximum eccentricity for planet $i$ results when the contributions from these two modes are in phase, yielding a value that is simply their sum $e_{i, \max }=e_{i 3}+e_{i 4}$. This is shown with a solid line for both planets. We compare these predictions with the results of $N$-body integrations by identifying the maximum osculating eccentricity of Mars 
and Earth observed in each simulation prior to the time when Jupiter and Saturn crossed their 2:1 MMR. These values are shown with filled circles.

For short migration timescales $\left(\tau \lesssim 10^{5} \mathrm{yr}\right)$, the initial eccentricities $\left(e_{i j} \simeq 0.005\right)$ dominate over contributions from the secular resonances. For longer migration timescales, the excitation of the resonances is more apparent. The $N$-body results always fall below the theoretical maximum and above the predicted amplitudes due to single modes. The time required to reach the maximum eccentricity of a two-mode system is the about half the beat period of two eigenfrequencies. For the $j=3$ and $j=4$ modes this is $\sim 1$ Myr. For $\tau \gtrsim 1$ Myr there is sufficient time in the simulation to realize the maximum eccentricity prior to the Jupiter-Saturn 2:1 crossing and the values measured from $N$-body simulations cluster tightly near the theoretical maximum. For $\tau \gtrsim 10^{5}$ yr the maximum eccentricities of both planets follow the $\tau^{1 / 2}$ scaling of Equation (37) and are within 0.005 (the initial amplitude) of the theoretical prediction. Clearly, the theory described above provides an accurate description of excitation during first passage through the $g_{3}-\nu_{5}$ and $g_{4}-\nu_{5}$ secular resonances.

We note that the predicted excitation of both modal eccentricity amplitudes of Mars $\left(e_{43}\right.$ and $e_{44}$ ) are comparable (see Figure 6(a)). For a given migration timescale $\tau$, the rate of frequency sweeping through the $g_{3}-\nu_{5}$ resonance is less (see $\left|d \nu_{5} / d a_{6}\right|$ in Figure 4), and the effective resonant width $\left(F_{35}\right)$ greater, than for sweeping through the $g_{4}-\nu_{5}$ resonance. Both factors contribute to the greater excitation of $C_{3}$ relative to $C_{4}$. Finally, the eigenvector components of Mars in the $j=3$ and $j=4$ modes are comparable in magnitude (i.e., $\left|v_{4,3}\right| \simeq\left|v_{4,4}\right|$, see Figure 1). The net effect is that for any migration timescale $(\tau)$, the eccentricity excitation of the $j=3$ component in Mars' eccentricity is actually greater than that of the $j=4$ mode (i.e., $e_{43} \simeq 1.1 e_{44}$, see Figure 6(a)). This appears in contrast to the observed eccentricity partitioning for Mars, where $e_{43}=0.020$ and $e_{44}=0.067$.

\subsection{Crossing the 2:1 MMR}

The increase in the eccentricities of Jupiter and Saturn as they diverge across their mutual 2:1 resonance might be communicated to the terrestrial planets. This change in the eccentricities and longitude of perihelia occurs over the circulation period of the 2:1 resonant argument $\left(\mathcal{O}\left(10^{3}\right)\right.$ yr). As this change occurs over a timescale much shorter than the secular periods, this eccentricity excitation might be viewed as a sudden change in system's mode amplitudes $C_{j}$ and phases $\beta_{j}$. Examination of the 2:1 MMR crossing finds that this impulsive event fairly purely excites the $C_{6}$ mode amplitude and that the excitation of the $C_{5}$ mode, is weak $\left(e_{55} \sim \mathcal{O}\left(10^{-3}\right)\right)$ and insufficient to explain its observed value (Morbidelli et al. 2009) 5 In general, this resetting of the giant planets' eccentricities should alter the entire system's mode amplitudes. However, in the observed solar

\footnotetext{
${ }^{5}$ The conference presentation of $\mathrm{Cuk}(2007)$ also highlighted this aspect of the 2:1 crossing.
} 


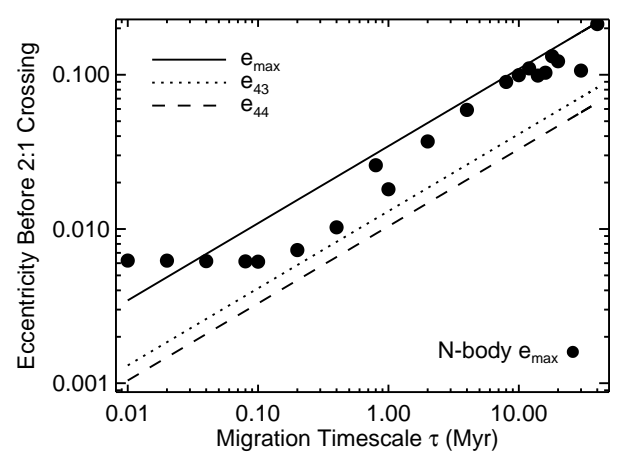

(a) Mars

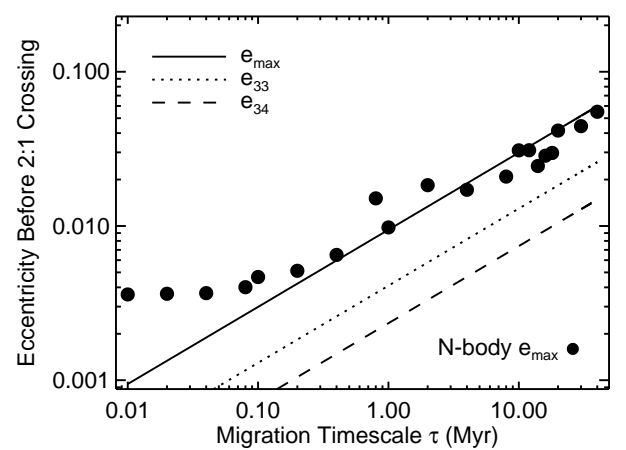

(b) Earth

Fig. 6. - Eccentricity of Mars $(i=4)$ and Earth $(i=3)$ excited during Jupiter and Saturn's approach to their 2:1 mean motion resonance as a function of the migration timescale $(\tau)$. The dotted and dashed lines indicate the prediction of resonant excitation of the $e_{i 3}$ and $e_{i 4}$ eccentricity amplitudes. The theoretical maximum eccentricity that might result is the sum of these two contributions and is shown with the solid line. The maximum osculating eccentricity observed in the simulations prior to 2:1 MMR crossing is shown with a filled circle. In both the linear theory and the $N$-body simulations the forcing eccentricity of Jupiter is about one-third the observed value (i.e., $e_{55} \approx 0.015$ ). The critical migration parameters $\hat{\tau}_{35}^{*}$ and $\hat{\tau}_{45}^{*}$ suggest migration timescales of $\tau=5$ and $\tau=30$ Myr can excite the $j=3$ and $j=4$ modes to their observed amplitudes.

system, the $j=6$ mode weakly contributes to the eccentricity evolution of the terrestrial planets (see the small values of $e_{i 6}$ in Table 1). So, the terrestrial eccentricity amplitudes $\left(e_{i j}\right.$, where $i, j=1--4)$ are modified by small amounts to accommodate an increase in the $j=6$ amplitude. On this basis, we suggest that the impulsive excitation of the $C_{6}$ mode amplitude is only weakly communicated to the terrestrial planets and its effect on the terrestrial $j=1-4$ modes is small relative to contributions from the $g_{j}-\nu_{5}$ secular resonances.

\subsection{The Second Crossing of the $g_{3}-\nu_{5}$ and $g_{4}-\nu_{5}$ Resonances}

The excitation obtained from the second crossing of the $g_{4}-\nu_{5}$ and $g_{3}-\nu_{5}$ resonances can also be predicted using Equation (37). For a given migration timescale the excitation of the $j=3,4$ resonances encountered inside of the Jupiter-Saturn 2:1 MMR are comparable to the excitation due to these secular resonances outside the $2: 1$.

Depending on the relative phasing between excitation from the first and second resonance crossings, these two contributions may interfere constructively or destructively and result in eccentricities larger or smaller than predicted for passage through a single resonance. Figure 7 shows examples from $N$-body simulations that illustrate both constructive and destructive interference 
due to successive passages through the $g_{3}-\nu_{5}$ and $g_{4}-\nu_{5}$ resonances as Jupiter and Saturn cross their 2:1 MMR.

In both examples the migration timescale $\tau$ is sufficiently long that the first secular resonance crossing near $t / \tau=0.4$ excites both $j=3$ and $j=4$ modes to amplitudes larger than their observed values. For the case shown in (a) the second passage through resonance results in a further increase in eccentricity amplitudes. In the second example (b), the contributions from the two resonances interfere destructively resulting in a final eccentricity state consistent with the observed terrestrial planets. This occurs despite a migration parameter greater than the critical value.

The latter result illustrates that Jupiter and Saturn's crossing of the 2:1 MMR can temporarily excite the eccentricity of Mars to values of 0.2 or larger. If Mars' orbit was excited in this way it may have facilitated the depletion of otherwise dynamically stable and unpopulated regions exterior to Mars' $a=1.7-2.0$ AU (Evans \& Tabachnik 2002; Bottke et al. 2010).

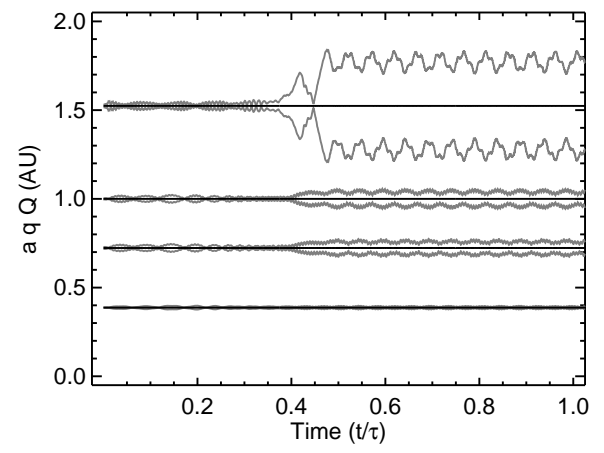

(a) $\tau=20 \mathrm{Myr}-$ Constructive Interference

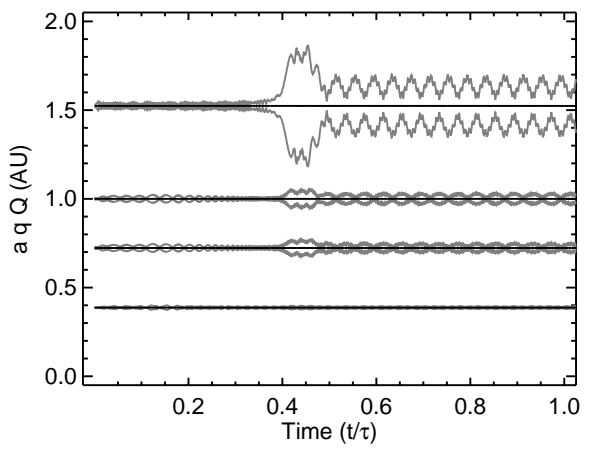

(b) $\tau=40 \mathrm{Myr}-$ Destructive Interference

Fig. 7.- Forced migration simulations with $\tau=20$ and 40 Myr. The evolution of the Jupiter and Saturn (not shown here) is similar to that in Figure 5. The black and gray lines represent the semimajor axes, pericenter, and apocenter distances, respectively. In (a) the contributions from crossing the secular resonances twice has an additive effect, resulting in a large final eccentricity for Mars. In (b) the excitation of Mars, Earth, and Venus due to two passages through the $g_{3}-\nu_{5}$ and $g_{4}-\nu_{5}$ resonances partially cancel and leave Mars, Earth, and Venus in a dynamical state similar to the observed one. Because of the smaller Jovian eccentricity amplitude $e_{55} \simeq 0.015$ the simulation in (a) and (b) correspond to migration parameters of 2.4 and $4.8 \mathrm{Myr}$, respectively.

For each simulation in this set of models we conducted an additional simulation to measure the eccentricity amplitudes $\left(e_{i j}\right)$ of the terrestrial planets via Fourier analysis. The FMFT-measured values for Earth $\left(e_{33}\right)$ and Mars $\left(e_{44}\right)$ are shown in Figure 8 with filled circles as a function of the migration timescale $\tau$. Using linear secular theory we have computed the eccentricity amplitude for each planet due to secular resonance crossing both interior and exterior to the 2:1 MMR. As expected the net effect of the contributions from these two resonances is stochastic and depends 
on the relative phase between them. Using Equation (28) as a distribution function we show the median eccentricity expected with a black solid line. The eccentricity amplitudes of the 25 th and 75th percentiles $\left(P_{25}\right.$ and $\left.P_{75}\right)$ are shown with dotted lines. Each line follows the $\tau^{1 / 2}$ scaling.

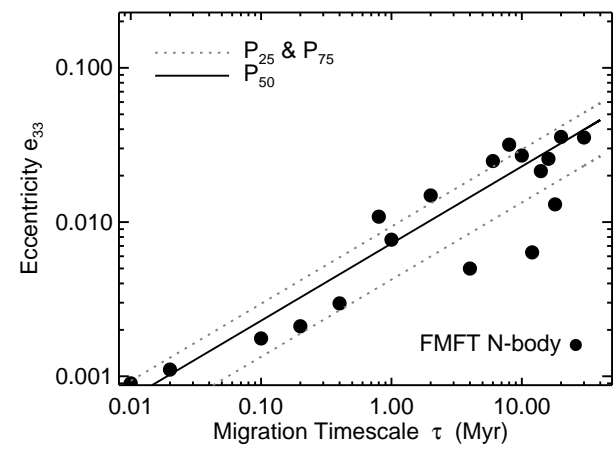

(a) Earth

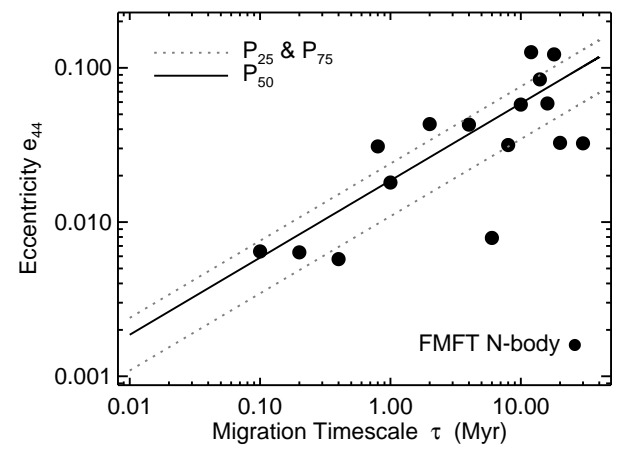

(b) Mars

Fig. 8.- FMFT-measured eccentricities for Earth $\left(e_{33}\right)$ and Mars $\left(e_{44}\right)$ are shown as a function of the migration timescale $(\tau)$. The scatter in these measured results is due to interference between contributions from multiple secular resonance passages. Using secular theory and the distribution function of Equation (28) we also show the eccentricities of the 50th (solid line), 25th, and 75th percentiles $\left(P_{25}\right.$ and $P_{75}$ dotted lines). For comparison the principal eccentricity amplitudes of Earth and Mars are $e_{33} \simeq 0.01$ and $e_{44} \simeq 0.07$, respectively (see Table 1).

Due to the stochastic influence of multiple resonance passages there is significant scatter in the eccentricities produced. However, about half of the FMFT-measured eccentricity amplitudes lie between the 25th and 75th percentile curves indicating that the results are in good accord with the distribution suggested by Equation (28) and demonstrate that the linear model effectively predicts characteristic levels of excitation and interference.

\subsection{Interference of Multiple Contributions}

If the $\nu_{5}$ frequency swept through resonances with both the $g_{3}$ and $g_{4}$ frequencies then the large amplitude of the $j=4$ mode relative to the $j=3$ mode requires explanation. Perhaps the relative amplitudes of the $j=3,4$ modes resulted from the interference between multiple contributions. Linear secular theory and our results above suggest that the final modal eccentricity amplitudes $e_{i j}$ can be calculated as the vector sum of multiple contributions (as in Equation 28). The $g_{3}-\nu_{5}$ and $g_{4}-\nu_{5}$ resonances are close together and are each encountered twice in rapid succession as Jupiter and Saturn diverge across their 2:1 MMR. These resonant contributions combine with any primordial amplitude that resulted from the planet formation epoch.

Studies of the gas free, late stage accretion of terrestrial planets have had difficulty simultane- 
ously accounting for the small eccentricities of Earth and Venus and the smallish masses of Mercury and Mars. Further, terrestrial planet formation simulations often produce planets with masses 0.7$1.0 M_{\oplus}$ with orbital eccentricities larger than Earth or Venus (see, e.g., Chambers \& Wetherill 1998; Agnor et al. 1999; Chambers 2001; O'Brien et al. 2006; Raymond et al. 2009) and suggest that a broad range of eccentricities might plausibly arise from accretion processes.

Here we examine the relative probability of eccentricity amplitudes combining to yield a dynamical state comparable to the observed one for the terrestrial planets. We consider the contributions to each mode individually below. We compute the final value of the $e_{33}$ eccentricity amplitude from the vector sum of an assumed initial amplitude (e.g., one resulting from formation) and two contributions from passage through the $g_{3}-\nu_{5}$ resonances on both sides of the 2:1 Jupiter-Saturn MMR. For a given value of the migration parameter $(\hat{\tau})$, the amplitudes of resonant contributions are determined using Equation 37. We then choose their relative orientations at random, combine them and compute the resultant eccentricity vector. For each value of the migration parameter this procedure is repeated $10^{4}$ times and the cases where the final eccentricity amplitude is similar to the observed state of terrestrial planets are counted.

When examining the results for the $j=3,4$ modes, we consider final eccentricity states with $e_{33}<0.02$ and $0.05<e_{44}<0.09$ to be "close" to the observed dynamical state of the terrestrial planets. While this definition is somewhat arbitrary, the first constraint is within a factor of two of the small $e_{33}$ amplitude, and the second is within $\simeq 30 \%$ of the observed $e_{44}$ amplitude (see Table 1 for observed values).

\section{Constraints on the Origin of $e_{33}$}

In Figure 9(a) we show the percent of cases that result in $e_{33}<0.02$ as a function of the migration parameter for small (0.01), modest (0.05), and large (0.10) initial modal eccentricity amplitudes. Note that the critical migration parameter for the $g_{3}-\nu_{5}$ resonances interior and exterior to the $2: 1$ are $\hat{\tau}_{35}^{*} \simeq 0.60$ and $0.33 \mathrm{Myr}$, respectively. For migration parameters larger than $0.7 \mathrm{Myr}$, crossing each $g_{3}-\nu_{5}$ resonance contributes a component larger than the observed amplitude and significant cancellation is required to achieve the observed small amplitude. For small initial eccentricity amplitudes (0.01), the percent of outcomes with $e_{33}<0.02$ decreases from $100 \%$, to about $20 \%$ as the migration parameter increases from 0.1 to $2.0 \mathrm{Myr}$.

When either the initial amplitude $\left(e_{33}=0.05,0.10\right)$ or resonant contributions are substantially larger than the observed $e_{33}$ value (e.g., for $\hat{\tau}>\hat{\tau}_{35}^{*}$ ), significant cancellation between all three contributions is required to produce a small final eccentricity. Figure 9(a) shows that strong cancellation and $e_{33}<0.02$ can happen, but is an infrequent occurrence for large initial eccentricity amplitudes $e_{33}=0.05,0.10$ or migration parameters greater than 2-5 Myr. On the other hand, the origin of the small $e_{33}$ amplitude is broadly consistent with a small primordial amplitude 


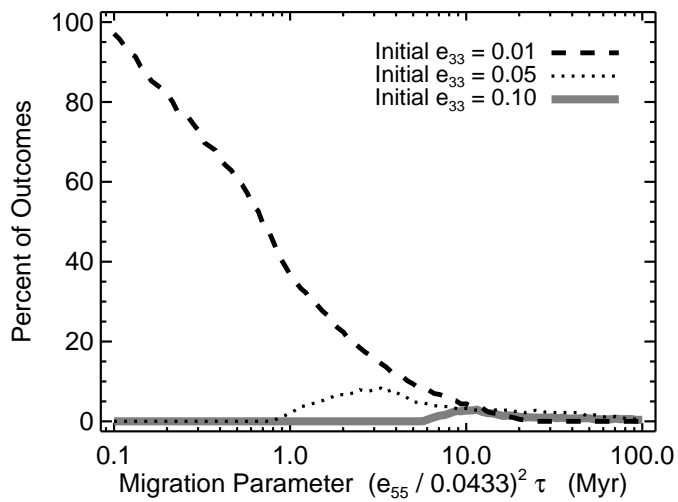

(a) Percent with $e_{33}<0.02$

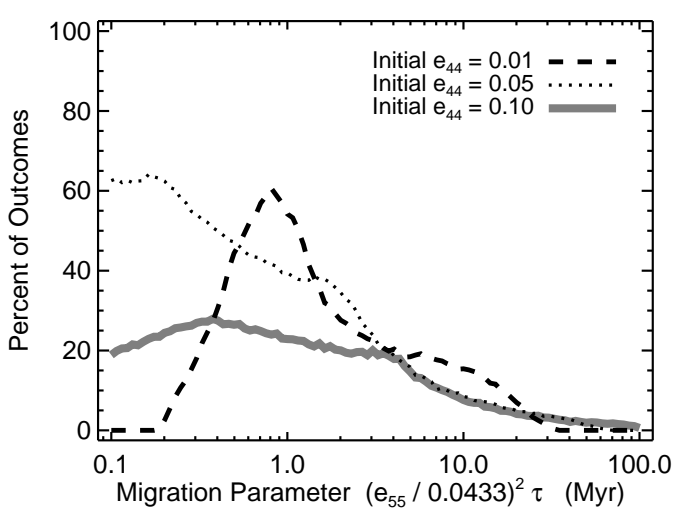

(b) Percent with $0.05<e_{44}<0.09$

Fig. 9.- Percent of outcomes where the $e_{33}$ and $e_{44}$ eccentricity amplitudes are close to those of the observed terrestrial planets are shown as a function of the migration parameter (Equation (35)). As Jupiter and Saturn migrate across the 2:1 MMR, the $g_{3}-\nu_{5}$ and $g_{4}-\nu_{5}$ resonances are each crossed twice, once on each side of the 2:1. Contributions from these resonances are combined with an assumed initial amplitude and the percentages of outcomes estimated using a Monte Carlo technique. Curves for different assumed initial eccentricities are shown.

(i.e., $\left.e_{33} \simeq 0.01\right)$ and migration parameters $\lesssim 1-2 \mathrm{Myr} 6$ It might also be explained by the cancellation of large resonant and initial contributions, but this becomes less likely when the contributions are substantially larger than the observed amplitude.

\section{Constraints on the Origin of $e_{44}$}

As above, we compute the resultant amplitude of the $j=4$ mode as being comprised of an assumed initial amplitude and contributions from both $g_{4}-\nu_{5}$ resonances. The critical migration parameters for the resonances interior and exterior to the $2: 1$ are $\hat{\tau}_{45}^{*} \simeq 3.8$ and $1.7 \mathrm{Myr}$, respectively. The percent of cases that produce a final eccentricity amplitude with $0.05<e_{44}<0.09$ is shown in Figure 9(b) as a function of the migration parameter for three different assumed initial eccentricities.

For small initial amplitudes $\left(e_{44}=0.01\right)$, this mode must be excited by the secular resonances to values near the observed amplitude. Resonant excitation from migration parameters $<0.2$ Myr is insufficient to perturb the $j=4$ mode to the observed state (see the dashed black line in Figure 9(b)). For migration parameters in the range of $0.5-1.0 \mathrm{Myr}$, about $50 \%$ of cases yield values

\footnotetext{
${ }^{6}$ Note this upper limit on the migration parameter $\hat{\tau}$ is larger than $\hat{\tau}_{35}^{*}$ listed in Table 2 as we are considering amplitudes larger than observed amplitudes of $e_{33}$ as acceptably close to the observed system.
} 
consistent the observed $e_{44}$ amplitude. As the migration parameter increases above $1.0 \mathrm{Myr}$, the percent of cases consistent with the observed value of $e_{44}$ decreases to about $5 \%$ at about 20 Myr.

When the initial eccentricity amplitude is larger (e.g., $e_{44}=0.05$ ), excitation from migration parameters $\hat{\tau}<0.4 \mathrm{Myr}$ is insufficient to alter the initial amplitude out of the acceptable range in more than $50 \%$ of cases (see the black dotted line in Figure 9(b)). Even with a large initial amplitude $e_{44}=0.10$, cancellation between the multiple contributions produces final amplitudes consistent with the observed one in $\gtrsim 20 \%$ of cases for a large range of migration parameters $(\hat{\tau} \simeq 0.1-2 \mathrm{Myr}$, see the gray solid line in Figure 9(b)).

The observed $e_{44}$ amplitude is sufficiently large that it can be achieved from initial amplitudes in the range of 0.01-0.1 and migration parameters $0.4-2$ Myr with a typical occurrence frequency of $20 \%-50 \%$ (or higher depending on specific parameter values and amplitudes).

\subsection{Implications}

The small observed $e_{33}$ amplitude provides a stronger constraint on giant planet migration than the $e_{44}$ amplitude. Specifically, the primordial, pre-migration $C_{3}$ mode amplitude must be less than or comparable to its current value (i.e., $e_{33} \lesssim 0.01$ ) and the migration parameter $\hat{\tau} \lesssim 2$ Myr to account for $j=3$ amplitude without requiring the low probability cancellation between large contributions.

If we consider the evolution of the $j=3$ and $j=4$ modes as independent, we can combine the analysis of the individual modes to ascertain the probability of these resonance producing a terrestrial system with both $e_{33}$ and $e_{44}$ close to their observed values as the product of the probability of two independent events. For initial amplitudes of $e_{33} \lesssim 0.01$ and $e_{44} \lesssim 0.05$ there is a reasonable range of migration parameters (0.6-1 Myr) where the constraints on both $e_{33}$ and $e_{44}$ can individually be satisfied in $\gtrsim 50 \%$ of cases. While our analysis is approximate, it suggests that

crossing the 2:1 MMR might produce a state similar to the observed eccentricity partitioning of the $j=3$ and $j=4$ terrestrial modes in $\simeq 25 \%$ of cases with these initial amplitudes and migration parameters.

\section{The $g_{2}-\nu_{5}$ and $g_{1}-\nu_{5}$ Secular Resonances}

For eccentricities of Jupiter and Saturn comparable to their current values as they passed through the $g_{2}-\nu_{5}$ and $g_{1}-\nu_{5}$ resonances, our secular theory indicates migration parameters $\hat{\tau} \gtrsim 0.05 \mathrm{Myr}$ and $0.7 \mathrm{Myr}$ will excite the terrestrial $j=2$ and $j=1$ modes to values larger than the observed ones. The order of magnitude difference in the critical parameters between these modes is primarily due to the larger width $\left(F_{25}\right)$ of the $g_{2}-\nu_{5}$ resonance and the smaller amplitude of the $j=2$ mode. A migration timescale of $0.05 \mathrm{Myr}$ is actually less than the period of the resonant 
frequency $(\approx 0.17 \mathrm{Myr})$. For such rapid evolution it is no longer appropriate to consider the secular mode amplitudes $\left\{C_{j}\right\}$ as effectively constant during migration.

We again explore the dynamics of passage through these resonances by conducting $N$-body simulations of forced giant planet migration with timescales that span the adiabatic secular $(\tau \simeq 1$ Myr) and non-adiabatic migration $(\tau \simeq 0.01 \mathrm{Myr})$ regimes. Migration timescales as short as $\tau \simeq 0.01$ Myr are not likely to result from smooth migration driven by the scattering of small bodies by Jupiter and Saturn. Such rapid migration might result from scattering with other planets or ice giants. In this sense our use of smooth migration with very short migration timescales may serve as a simple proxy for the rapid non-adiabatic evolution driven by giant planet scattering.

\subsection{Initial Conditions}

We again isolate the influence of secular resonances by constructing initial conditions where the terrestrial mode amplitudes are very small, the giant planets' modal amplitudes are near their present values, Jupiter's semimajor axis is fixed at 5.2 AU, and Saturn's initial semimajor axis of 8.5 AU places the $\nu_{5}$ frequency between the $g_{3}$ and $g_{2}$ frequencies. We prepared this initial condition using a series of orbital integrations. First, we performed an integration starting with only Jupiter and Saturn with their observed orbits and slowly forced Saturn to migrate inward to a semimajor axis of 8.5AU. Because the forced migration is slow relative to the secular timescale, the $D_{5,6}$ modal amplitudes are effectively constant during migration to the new orbits. The terrestrial planets were then added to the system and we conducted a second integration applying slow eccentricity damping to decrease the terrestrial mode amplitudes. The resulting state of the Jupiter-Saturnterrestrial planet system was then used as the initial condition in the numerical experiments that follow. FMFT analysis of this initial condition finds the terrestrial eccentricity amplitudes with $e_{i j} \lesssim 0.005$ and the $e_{55}$ amplitude of Jupiter very near its current value (i.e., $e_{55} \simeq 0.0435$ ). Thus, for this set of simulations $\hat{\tau}=\tau$.

\subsection{Results}

Figure 10 shows the eccentricity evolution of the terrestrial planets as Saturn migrates from 8.5AU $\rightarrow 9.5 \mathrm{AU}$ with an exponential timescale of $\tau=2$ Myr. The $g_{2}-\nu_{5}$ and $g_{1}-\nu_{5}$ resonances are crossed at about $t / \tau=0.3$ and 0.6 when Saturn is near 8.7 and 8.9 AU, respectively. Excitation of both terrestrial eigenmodes is evident in the relative timing of the maximum and minimum eccentricity observed in the orbits of Mercury, Venus, and Earth. Mercury's osculating eccentricity reaches a maximum when contributions from both the $j=1$ and $j=2$ modes are in phase. For this same orientation between modes, the eigenvector signatures for Earth and Venus are anti-aligned (see Figure11) resulting in cancellation between modal contributions and the osculating eccentricities of Earth and Venus simultaneously reach a minima. For this cancellation to yield eccentricities near 
zero, the eccentricity amplitudes from each mode must be comparable. In agreement with secular theory, the eccentricity amplitudes of Mercury, Venus, and Earth are each driven to values in excess of the present-day values. Mars is only weakly coupled to the other terrestrial planets through the $j=1,2$ modes (see Figure 1) and is minimally excited via these resonances.

Following each migration simulation we conducted a separate orbital integration and performed FMFT analysis of the resulting dynamical state. The modal eccentricity amplitudes $\left(e_{i j}\right)$ for Mercury, Venus, and Earth are shown in Figure 11 as a function of the migration timescale $(\tau)$. The predicted excitation of the $j=1,2$ modes are shown as solid and dashed lines, respectively. Also the FMFT-measured eccentricity amplitudes for the $j=1,2$ modes are shown with diamonds and filled circles, respectively.

For migration timescales $\tau=0.1 \rightarrow 0.4 \mathrm{Myr}$, the eccentricity amplitudes closely match the predictions of secular theory and follow the $\tau^{1 / 2}$ scaling. For Mercury the excitation of $e_{11} \simeq 0.20$ requires $\tau=0.6 \mathrm{Myr}$. Note the $j=2$ component of Mercury's eccentricity is excited to half that of the $j=1$ mode $\left(e_{12} \approx 0.5 e_{11}\right)$ for any timescale $\tau$. For slower migration, Mercury may be excited to very large eccentricity due to the combined effect of both resonances. In these cases general relativity and higher order effects conspire to increase Mercury's precession rate and the value of the $g_{1}$ eigenfrequency (Laskar 2008). For $\tau=0.3 \rightarrow 1.0 \mathrm{Myr}$, the FMFT measurement of the $g_{1}$ eigenfrequency increases from $5.84^{\prime \prime} \mathrm{yr}^{-1} \rightarrow 6.3^{\prime \prime} \mathrm{yr}^{-1}$. This tuning of the $g_{1}$ toward the $g_{2}=7.43^{\prime \prime} / \mathrm{yr}$ alters the structure of both eigenvectors. As two eigenfrequencies converge, their eigenvector signatures become increasingly similar (see detailed discussions of this issue in Ward 1981). As the value of $g_{1}$ increases toward the $g_{2}$, the magnitude of the components of Earth and Venus in the $j=1$ eigenvector increase and those of Mercury slightly decrease. The $j=2$ eigenvector evolves in a complementary fashion. The component of Mercury in the $j=2$ mode $\left(v_{12}\right)$ increases while the components of Venus and Earth $\left(v_{22}\right.$ and $\left.v_{32}\right)$ decrease. In this case, the modest discrepancies in FMFT-measured eccentricity amplitude and secular theory in Figure 11 result from changes in the eigenfrequencies and eigenvector signatures. We note that these effects become important at eccentricities well above the observed values in the present solar system.

For migration timescales $\tau<0.1 \mathrm{Myr}$, the entire epoch of migration may occur in less than a precession cycle. Such migration is not slow with respect to the $\nu_{5}$ frequency. Since the eccentricity amplitudes of the terrestrial planets were all $e_{i j} \lesssim 0.005$ prior to migration, Figure 11 shows that rapid non-adiabatic giant planet migration can also contribute to the eccentricity amplitudes and AMD in the terrestrial modes. For the $j=2$ mode, the eccentricity amplitudes of $e_{12}=0.052$, $e_{22}=0.033$, and $e_{32}=0.025$ for Mercury, Venus, and Earth are systematically achieved and appear independent of the migration timescale. These values correlate with the planet's distance from the $\nu_{5}$ resonance in the present-day solar system and are likely related to the forced eccentricity in the region. These values are also greater than the observed values for the $j=2$ eccentricity amplitudes of the terrestrial planets.

For the fastest migration timescale explored above, this change in eccentricity amplitudes re- 

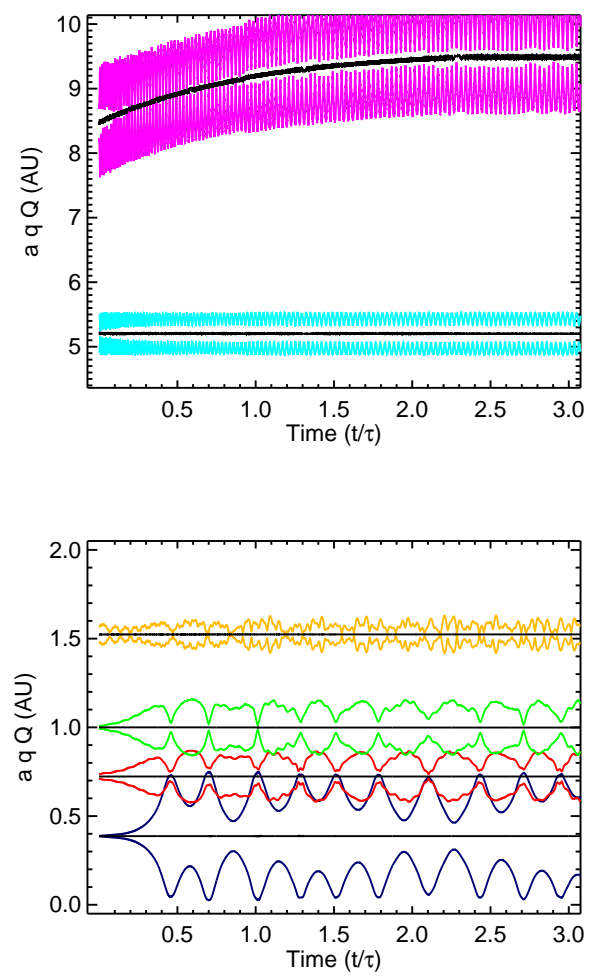

Fig. 10. - Forced migration simulation with $\tau=2.0$ Myr. The semimajor axes of the Jupiter and Saturn and the terrestrial planets are shown with a black solid line. The pericenter and apocenter are shown in gray (colored in online version). 


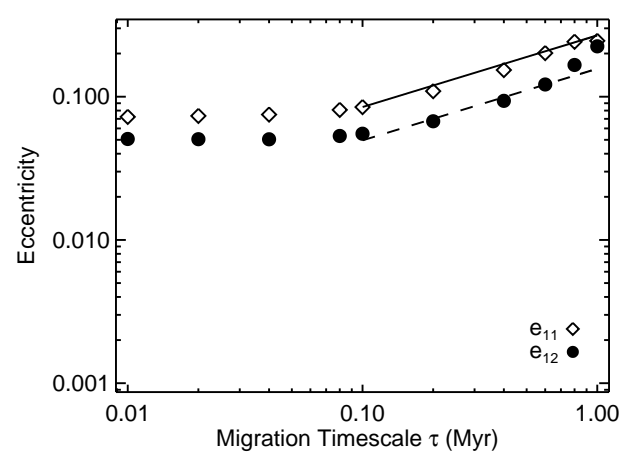

(a) Mercury

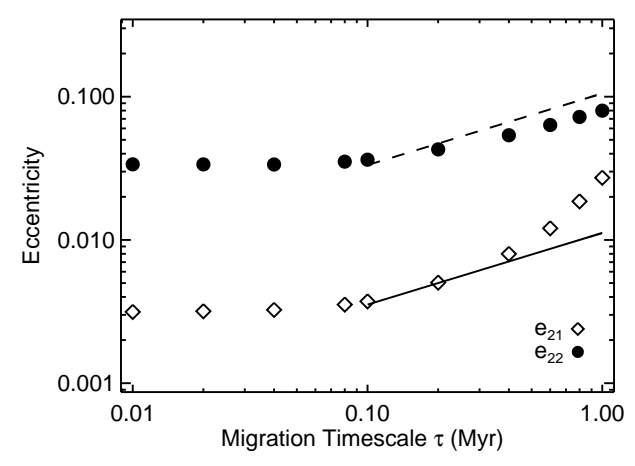

(b) Venus

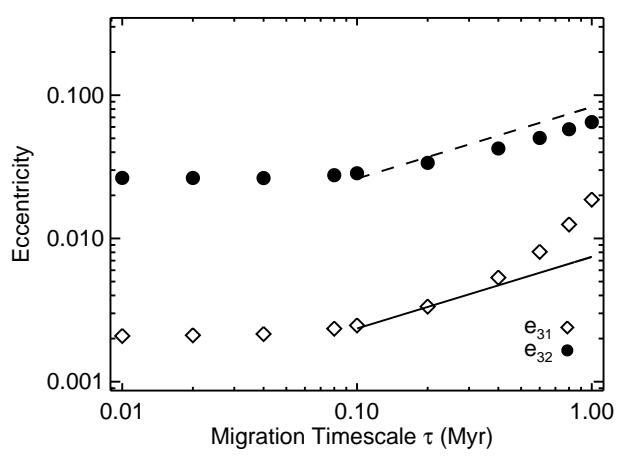

(c) Earth

Fig. 11. - Final modal eccentricity amplitudes $\left(e_{i j}\right)$ of the terrestrial planets as a function of the giant planet migration timescale $(\tau)$. The symbols indicate FMFT measurements of the amplitude from $N$-body simulations and the lines indicate the prediction of secular theory for the excitation of the $j=1,2$ modes in each planet (Equation (37)). Results for the $j=1$ mode are shown with solid lines and open diamonds and results for $j=2$ mode are shown with dashed lines and filled circles.

sults from a single rapid jump from one orbital state of Jupiter and Saturn to another. However, giant planet migration due to scattering between the planets would involve several to many stochastic jumps in semimajor axes and modal amplitudes (Morbidelli et al. 2009). Repeated scattering events among the giant planets may induce stochastic and diffusive exchange of AMD between the terrestrial and giant planet mode amplitudes. If the eccentricities shown in Figure 11 are indicative of the size of the steps, then this type of evolution may also perturb the terrestrial system to eccentricities larger than the observed values. As in the case of slower planetesimal-driven migration, selective cancellation between multiple contributions may be required for the terrestrial planets to emerge from this type of giant planet evolution with an eccentricity state resembling the observed one, even if the initial $j=2,3$ mode amplitudes are small. Brasser et al. (2009) show an $N$-body 
simulation result that illustrates this type of evolution. A systematic exploration of the diffusion of AMD between the giant and terrestrial planets during an epoch of giant planet scattering is needed to more generally evaluate the influence this style of giant planet migration has on the terrestrial planets.

\subsection{Interference of Resonant and Primordial Amplitudes}

There is about an order of magnitude difference between the critical migration parameters for the $j=2$ and 1 modes $\left(\hat{\tau}_{25}^{*}=0.05 \mathrm{Myr}\right.$ and $\hat{\tau}_{15}^{*}=0.7 \mathrm{Myr}$, see Table 21) with both values shorter than migration timescales suggested for planetesimal-driven migration by at least an order of magnitude. Could cancellation between the resonant contributions yield a dynamical state consistent with the terrestrial planets for migration timescales of 2-10 Myr? To evaluate this hypothesis we model the addition of multiple contributions to the $j=2,1$ modes using the Monte Carlo technique described in Section 4.5. Because the $g_{2}-\nu_{5}$ and $g_{1}-\nu_{5}$ resonances are crossed once during the last $\sim 1 \mathrm{AU}$ of divergence between Jupiter and Saturn, the final mode amplitude results from just two contributions, the secular resonant one and the primordial amplitude. When comparing results with the terrestrial planets, we consider "close" to the observed dynamical state to mean $e_{22}<0.03$ and $0.12<e_{11}<0.23$ for the $j=2$ and $j=1$ modes, respectively. As above, these ranges are somewhat arbitrary, but are comparable to the observed $e_{22}=0.02$ amplitude and within $30 \%$ of the observed $e_{11}=0.18$ amplitude.

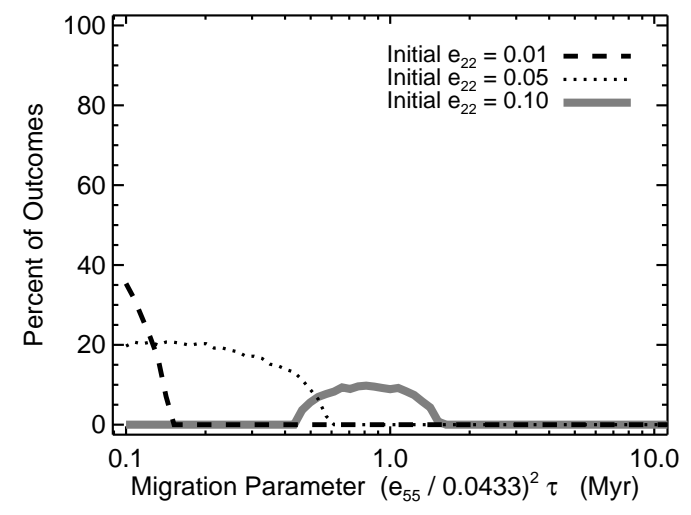

(a) Fraction with $e_{22}<0.03$

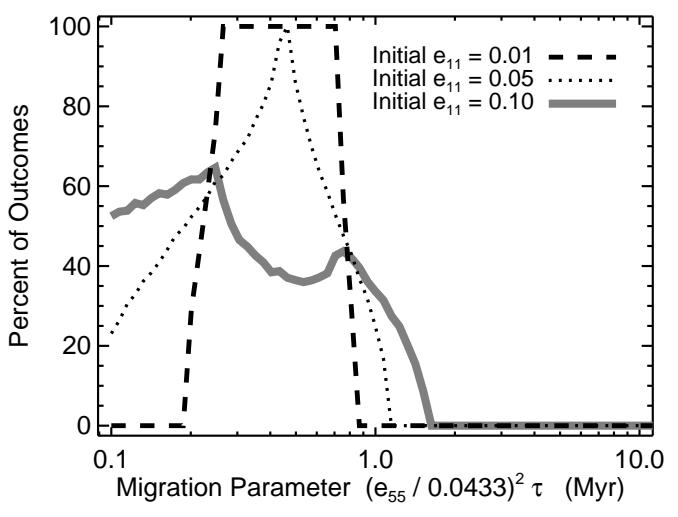

(b) Fraction with $0.12<e_{11}<0.23$

Fig. 12.- Percent of outcomes where the $e_{22}$ and $e_{11}$ eccentricity amplitudes are close to the observed dynamical state of the terrestrial planets are shown as a function of the migration parameter (Equation (35)). Contributions from the $g_{2}-\nu_{5}$ and $g_{1}-\nu_{5}$ resonances are combined with an assumed initial amplitude as in Figure 9. Curves for different assumed initial eccentricities are shown. 
Figure 12 shows the percent of outcomes consistent with the observed $e_{22}$ and $e_{11}$ as a function of the migration parameter for several different assumed primordial eccentricity amplitudes. Cancellation can produce systems with $e_{22}<0.03$ for migration parameters larger than the critical one (see, e.g., Figure 12(a)), but the requisite destructive interference requires primordial and resonant contributions of comparable size. Also, the probability of the required cancellation decreases as the size of contributions (and migration parameter) increases. For example, migration parameters of 1 Myr contribute a vector of about 0.10 in magnitude to $e_{22}$ (see Equation (37 and Table 22). Combining this with an initial eccentricity amplitude of 0.10 will produce a sufficiently small final value of $e_{22}$ in $\lesssim 10 \%$ of cases.

Figure 12(b) shows results for the $e_{11}$ amplitude. Cancellation with an initial $e_{11}$ amplitude generally broadens the range of migration parameters that are able to produce systems close to the observed state of the terrestrial planets. However, even large initial eccentricities of 0.10 do not permit migration parameters much larger than $1 \mathrm{Myr}$, and are not consistent with migration timescales suggested for planetesimal migration, unless the amplitude of the $e_{55}$ amplitude is lower than its observed value as these resonances are encountered.

Recall that the $N$-body simulations with migration timescales $\tau=0.01 \mathrm{Myr}$ are fast with respect to the secular timescale. Also for very fast migration the resulting $e_{11}$ and $e_{22}$ amplitudes are comparable to those obtained by migration parameters $\hat{\tau} \simeq 0.1 \mathrm{Myr}$. We use this level of excitation to estimate the minimum forcing expected during instability-driven migration (i.e., planet-planet scattering). For example, Figure 12 shows that for modest initial eccentricity amplitudes (i.e., $e_{11} \simeq$ $\left.e_{22} \simeq 0.05\right)$ and fortuitous interference between primordial and resonant contributions, both the large observed value of $e_{11}$ and the small observed value of $e_{22}$ might be produced in $\sim 10 \%$ of cases for migration parameters of 0.10-0.4 Myr. This fast exponential migration modeled here might be thought of as the rapid divergence of Jupiter and Saturn by a single strong scattering event, or one large jump in semimajor axis to the observed orbit. When Jupiter and Saturn experience multiple scattering events the forcing of the terrestrial planets may be greater and the success rate in producing the observed state of the inner planets lower.

Because the $e_{11}$ modal amplitude is about an order of magnitude larger than $e_{22}$ and both are excited in comparable ways by the sweeping $\nu_{5}$ resonance (see Equation (37), Table 2, and Figure 11), it is difficult to explain their very different amplitudes as a natural outcome of planetary migration, without invoking strong interference between multiple contributions or additional processes.

\section{Discussion}

Using a modified secular theory we have calculated how the $\nu_{5}$ and $\nu_{6}$ eigenfrequencies of the Jupiter-Saturn system evolve as these planets diverge from just outside their mutual 3:2 mean mo-

tion resonance to their observed orbits. Using the subgroup approximation of Ward (1981) we have 
identified six secular resonances between the $\nu_{5}$ frequency and one of the terrestrial eigenfrequencies $g_{j=1-4}$ during this divergent migration.

Based on analytic arguments we combined the Jovian eccentricity amplitude $\left(e_{55}\right)$ and orbital migration timescale $(\tau)$ into a single migration parameter $\hat{\tau}=\left(e_{55} / 0.0433\right)^{2} \tau$ that encapsulates the forcing strength experienced by the terrestrial planets via secular resonances with the $\nu_{5}$ frequency. We used this model to identify the critical values of the migration parameter required to excite the terrestrial eccentricity eigenmodes to their observed amplitudes. We validated the analytic secular model with $N$-body simulations and showed that linear secular models can account for the fundamental character and scaling of the eccentricity excitation experienced by the terrestrial planets via these secular resonances. We then used this model and the observed modal amplitudes of the terrestrial planets to constrain the migration of Jupiter and Saturn when these secular resonances were encountered.

\subsection{Evaluating Model Assumptions}

To clarify the dominant processes at work, we have made a number of simplifying assumptions. First, we have neglected the influence of Uranus and Neptune. The distant locations and relatively small mass of the ice giants render them weak perturbers of Jupiter and Saturn. Their omission from the secular model does not significantly modify the $\nu_{5}$ and $\nu_{6}$ frequencies of Jupiter and Saturn or those of the terrestrial planet subsystem (e.g., $\Delta g_{1} / g_{1} \sim \mathcal{O}\left(10^{-3}\right)$ ).

Similarly, we have neglected any modification of the system's eigenfrequencies that results from interaction with the planetesimal disk fueling migration. The modification of the $\nu_{5}$ and $\nu_{6}$ frequencies by the planetesimal disk is a function of the disk mass and location. When considering the late migration of Jupiter and Saturn, the planetesimal disk must have survived for at least the 30-100Myr required to form the terrestrial planets. Bodies with orbits near Jupiter and Saturn have a median dynamical lifetime of about 6-9 Myr (Tiscareno \& Malhotra 2003; Bailey \& Malhotra 2009). This suggests that the Jupiter-Saturn region was largely devoid of planetesimals by the time the terrestrial planets were completely assembled (see, e.g., Gomes et al. 2005). A planetesimal disk exterior to Jupiter and Saturn with mass comparable to that of Uranus and Neptune (i.e., $\sim 30 M_{\oplus}$ ) affects the $\nu_{5}$ and $\nu_{6}$ frequencies by an amount comparable to that of the ice giant planets and can be similarly neglected.

For rapid migration $(\tau<1-2 \mathrm{Myr})$, a large fraction of the planetesimal disk must be on crossing orbits with Jupiter and Saturn. To roughly estimate the effect of this disk on the giant planet eigenfrequencies we treat it as a single $10 M_{\oplus}$ planet between the gas giants. This increases both the $\nu_{5}$ and $\nu_{6}$ frequencies by $\simeq 20 \%$. However, this contribution decays as migration proceeds and the planetesimal disk is depleted. We suggest that these deviations in the $\nu_{5}$ frequency are not likely to prevent resonances with the terrestrial modes or grossly modify the factors that determine the excitation experienced by the inner planets. 
In exploring the problem we held Jupiter's semimajor axis constant to reduce the number of model parameters. More generally, the configuration of the planetary system when secular resonances occur depends on the semimajor axes of all the planets. So, the examples shown and values listed in Table 2 are not unique. Self-consistent $N$-body simulations of planetesimal-driven migration of the solar system typically show Jupiter to migrate inward a few tenths of an AU and Saturn migrating outward a few AU on the same timescale (e.g., Hahn \& Malhotra 1999; Gomes et al. 2004).

We have used the secular analytic model to examine how Jupiter's migration affects the planetary configuration where resonances occur and the critical migration parameter for each resonance. Considering Jupiter with an initial semimajor axis of 5.4 AU, Saturn starting just outside the 3:2 MMR with Jupiter, and both planets migrating to their observed orbits, all six of the secular resonances reported in Table 2 are encountered, albeit with slightly different values of the planetary semimajor axes, resonant frequencies, and critical migration timescales. While the $\nu_{5,6}$ frequencies are strongly affected by the orbital divergence of Jupiter and Saturn, the effect of Jupiter's inward migration of $\sim 0.2 \mathrm{AU}$ on the terrestrial eigenfrequencies $\left(g_{1-4}\right)$ is small and only slightly larger than the effect of Saturn's outward migration (see, e.g., Figure 4). Consequently, the orbital period ratio between Saturn and Jupiter $\left(P_{S} / P_{J}\right)$ at a particular $g_{j}-\nu_{5}$ secular resonances is nearly independent of Jupiter's semimajor axis, or whether it is migrating. This allows the period ratio to be used as a single independent variable to identify secular resonances (see, e.g., Brasser et al. 2009) and for comparison with scenarios where both gas giants are migrating.

Further, the secular model shows that the frequency gradient at resonance due to Jupiter $\left(\left|d\left(g_{j}-\nu_{5}\right) / d a_{5}\right|\right)$ is comparable to that of Saturn $\left(\left|d\left(g_{j}-\nu_{5}\right) / d a_{6}\right|\right)$. In this situation, the planet with the greater migration rate (i.e., $d a / d t$ ) most strongly determines the level of terrestrial excitation and the critical migration parameter (see Equation (36) ). Due to its larger mass Jupiter tends to be less mobile and its planetesimal-driven migration rate generically slower than Saturn's. Consequently, Saturn's migration rate most strongly determines excitation of the inner solar system. Finally, for the cases where Jupiter migrates inward a few tenths of an AU, the critical migration parameters vary from those listed in Table 2 by $20 \%-30 \%$.

In total, the various assumptions we have made to simplify the model and its presentation modestly affect the secular system. We consider the critical migration parameters listed in Table 2 as broadly representative of many plausible migration scenarios suggested for the solar system.

\subsection{Constraints on the Migration of Jupiter and Saturn}

As Jupiter and Saturn diverge across their mutual 2:1 MMR they encounter the $g_{3}-\nu_{5}$ and $g_{4}-\nu_{5}$ secular resonances on both sides of the resonance (i.e., for $P_{S} / P_{J} \simeq 1.97-2.03$ ). Due to several factors, including the small amplitude of the $j=3$ terrestrial mode, the $g_{3}-\nu_{5}$ resonances near the 2:1 MMR of Jupiter and Saturn constrain the giant planet migration parameter to satisfy 
$\hat{\tau} \lesssim \hat{\tau}_{35}^{*}=0.60$ Myr. Following the 2:1 crossing, the $g_{2}-\nu_{5}$ and $g_{1}-\nu_{5}$ resonances are encountered as Jupiter and Saturn diverge outside their 2:1 MMR (when $P_{S} / P_{J} \simeq 2.1-2.2$, see Table 2). In a similar manner the migration history considered must satisfy $\hat{\tau} \lesssim \hat{\tau}_{25}^{*}=0.05 \mathrm{Myr}$ when $P_{S} / P_{J} \simeq 2.1$ and $\hat{\tau} \lesssim \hat{\tau}_{15}^{*}=0.68 \mathrm{Myr}$ when $P_{S} / P_{J} \simeq 2.2$. We note that greater values of the migration parameters may also produce acceptable states for secular resonances with the terrestrial modes $(j=1-4)$. However, achieving agreement with the observed terrestrial system relies on cancellation of multiple contributions (see, e.g., Figures 7 and 8 ). When the magnitudes of all contributions are known, we have shown that the probability of producing a particular eccentricity state can be estimated using analytical arguments (see Equation (28), Figures 9 and (12). We have also shown that the probability of yielding the required cancellation between multiple contributions decreases significantly when the critical migration parameter $\hat{\tau}_{j 5}^{*}$ is exceeded by a factor of two to three. With these caveats we suggest that these values represent characteristic constraints on the migration of Jupiter and Saturn due to passage through these secular resonances. Further, because the solar system's giant planets migrate as a coupled system, constraints on the migration of Jupiter and Saturn should be considered as indirect constraints on the formation and migration of Uranus and Neptune and the evolution of the giant planet system as a whole.

For each $g_{j}-\nu_{5}$ secular resonance identified, planetesimal-driven migration of the giant planets (i.e., $\tau \simeq 5-20 \mathrm{Myr}$ ) with the Jovian eccentricity comparable to the present value would excite the terrestrial system to eccentricities and modal amplitudes well beyond their observed values. The constraints on giant planet migration imposed by these secular resonances and the dynamical structure of the terrestrial planets may be satisfied in several ways.

First, if the giant planets migrated with eccentricity amplitudes comparable to their present day values the strong forcing of the terrestrial planets via the secular resonances may have interfered destructively with an initial amplitude of the $j=1-4$ modes (e.g., from the accretion epoch). This could deposit the terrestrial planets in a dynamical state similar to the observed one. The general scenario requires the cancellation of two large components and we have shown that this is accordingly of low probability (see also Brasser et al. 2009).

Using the resonant excitation model and geometric arguments we can identify additional constraints required on this type of evolution. For cancellation to be viable, the initial and resonant contributions must be of a similar size and the excitation from resonance crossing necessarily less than an amount that would render the terrestrial subsystem unstable. For the $g_{3}-\nu_{5}$ and $g_{4}-\nu_{5}$ secular resonances encountered near the 2:1 MMR, the terrestrial planets are driven to crossing orbits if the migration timescale of Jupiter and Saturn is $\tau \gtrsim 10 \mathrm{Myr}$ and the $e_{55}$ has the present-day value. For the $g_{2}-\nu_{5}$ and $g_{1}-\nu_{5}$ resonances, $\hat{\tau} \gtrsim 2 \mathrm{Myr}$ leads to crossing orbits and instabilities (see, e.g., Figure 101). This latter constraint indicates that planetesimal-driven migration through the $g_{2,1}-\nu_{5}$ secular resonances is not viable unless the Jovian eccentricity amplitude $e_{55}$ is significantly smaller than the observed value.

Second, if the Jovian eccentricity $\left(e_{55}\right)$ was smaller during migration, then longer timescales 
might be permissible. For $\tau \sim 10 \mathrm{Myr}$, the $g_{3}-\nu_{5}$ secular resonance constrains the Jovian eccentricity amplitude $e_{55} \lesssim 0.011$, or about a quarter of the current value, when Jupiter and Saturn migrated across the 2:1 MMR. This eccentricity is comparable to those typical of models of planetesimal-driven migration. However, since it is less than the observed $e_{55}=0.0433$ value it also requires that the $D_{5}$ mode amplitude be excited later via some other process. Morbidelli et al. (2009) examined the origin of the $D_{5}$ mode amplitude in detail and found that repeated planetplanet scattering between Jupiter or Saturn and an ice giant planet may explain the observed $D_{5}$ amplitude and account for its excitation via an instability in the giant planets' orbits after the 2:1 MMR of Jupiter and Saturn is crossed. This analytic result is consistent with the numerical simulations of Brasser et al. (2009) and the conclusions drawn from them. Using the secular model we have also shown for initial eccentricity amplitudes $e_{33} \lesssim 0.01$ and $e_{44} \lesssim 0.05$ the eccentricity partitioning of the $j=3$ and $j=4$ terrestrial modes can be expected with a roughly $25 \%$ probability for migration parameters in the range $\hat{\tau}=0.6-1.0$ Myr. Again, these values are broadly consistent with those of planetesimal-driven migration.

When examining the $g_{2}-\nu_{5}$ and $g_{1}-\nu_{5}$ resonances, a Jovian modal eccentricity amplitude of $e_{55} \lesssim 0.003$ is required to allow a planetesimal-driven migration timescale of $\tau \simeq 10 \mathrm{Myr}$ through these resonances without exciting the $j=2$ terrestrial mode amplitude beyond its observed value. This value of $e_{55}$ is quite small, and again requires the late excitation of the Jovian eccentricity (e.g., via scattering with $\mathrm{a} \gtrsim 10 M_{\oplus}$ body to excite the $D_{5}$ amplitude during the last $0.5-0.8 \mathrm{AU}$ of divergent migration between Jupiter and Saturn; Morbidelli et al. 2009).

Third, rapid, stochastic migration in large steps in semimajor axis is also possible when the system of planets is unstable and planet-planet scattering ensues. Such global instabilities may allow secular resonances to be jumped over and the whole migration epoch to be much shorter than suggested by planetesimal-driven migration. (Thommes et al. 1999; Tsiganis et al. 2005; Morbidelli et al. 2007; Batygin \& Brown 2010). If such rapid migration was driven by scattering between planets, this process must cause the orbits of Jupiter and Saturn to diverge. For Saturn to move outward it must scatter an ice giant inward. Similarly for Jupiter to move inward, it must scatter an ice giant outward. This style of instability-driven migration might allow the $\nu_{5}$ and $\nu_{6}$ secular resonances to move quickly through the inner solar system. $N$-body simulations of this process by (Brasser et al. 2009) suggest that it is possible that the terrestrial planets might emerge from this style of planet migration with orbits comparable to their observed ones.

On the other hand, our simulations with very fast migration $(\tau<0.2 \mathrm{Myr})$ indicate that the $j=2$ terrestrial mode can be excited to amplitudes in excess of the observed values. If the $D_{5}$ mode amplitude originated via repeated planet-planet scattering (Morbidelli et al. 2009) it appears plausible that instability-driven giant planet migration may perturb the terrestrial planets to an overly excited state. In this regard, additional work assessing the relative frequency of characteristic outcomes (e.g., those similar to the observed state) would be welcome.

Finally, if planetary migration was rapid as suggested by the constraints of these secular reso- 
nances $(\tau \lesssim 5-10 \mathrm{Myr}$ ), then entire epoch of giant planet formation and migration, could have been largely complete before terrestrial planet accretion was finished. In this case the dynamical structure of the inner solar system was determined later (e.g., by processes related to accretion and evolution) and the formation timescale of the terrestrial planets (i.e., 30-100 Myr) constrains the timing by which giant planet formation and migration was largely complete. Recently, Walsh \& Morbidelli (2011) have investigated the effect of the early planetesimal-driven migration of Jupiter and Saturn on terrestrial planet formation. Their $N$-body accretion simulations with a migration timescale of $\tau=5$ Myr for Jupiter and Saturn produce terrestrial planet systems with AMDs comparable to the observed terrestrial planets. While several open problems of terrestrial planet formation persist in these simulations (e.g., explaining the small mass of Mars and the clearing and dynamical structure of the asteroid belt), these results suggest that effects of the sweeping $\nu_{5}$ resonance through the terrestrial region may be tempered by terrestrial accretion dynamics.

\subsection{Implications for the LHB}

In the Nice model explanation of the LHB (Gomes et al. 2005), an instability among the giant planets is initiated by Jupiter and Saturn crossing their mutual 2:1 MMR. This instability may be delayed by the very slow divergent migration of Jupiter and Saturn of a few tenths of an AU toward the 2:1 MMR over 600-700 Myr. This slow migration roughly corresponds to exponential migration timescales of $\tau \gtrsim 3 \times 10^{9} \mathrm{yr}$. The secular resonant model presented here predicts that crossing the $g_{3}-\nu_{5}$ and $g_{4}-\nu_{5}$ resonances this slowly requires the Jovian eccentricity amplitude $e_{55} \lesssim \mathcal{O}\left(10^{-4}\right)$ to leave the terrestrial planets in a state comparable to the observed one. A modestly larger Jovian eccentricity $\left(e_{55} \gtrsim 0.001\right)$ may perturb the terrestrial planets to crossing orbits and drive a global instability of the terrestrial system.

Requiring such small values of the Jovian eccentricity is a very strong constraint on the evolution of the gas giants and we interpret this as an indication that the slow divergent migration of Jupiter and Saturn toward the 2:1 MMR is not generally consistent with the coexistence and dynamical structure of the terrestrial planets. As a result, the specific LHB initiating scenario described in Gomes et al. (2005) appears unlikely. Brasser et al. (2009) have drawn similar conclusions based on the results of $N$-body simulations.

If the LHB resulted from the large scale migration of the giant planets, then an instability and giant planet scattering may be required to avoid strongly perturbing the terrestrial planets via secular resonances (Brasser et al. 2009). Alternate modes of triggering a late instability (e.g., Uranus and Neptune's divergent migration across a mean motion resonance) are possible and may offer viable alternative modes of initiating the LHB (Morbidelli et al. 2007).

However, the $j=5$ mode of Jupiter and Saturn has components in terrestrial planets comparable to those of $j=2,3$ terrestrial modes (see, e.g., Table 1). If giant planet scattering is responsible for exciting the $j=5$ mode amplitude to the observed value, then this excitation may 
also be communicated to the terrestrial planets (e.g., stochastic diffusion of AMD between $j=5$ mode and the terrestrial $j=1-4$ modes). Our simulations of very fast migration (i.e., $\tau \lesssim 0.10$ Myr) suggest that excitation of the terrestrial planets via this process is likely, but more work to examine the net exchange of AMD between the terrestrial and giant planet systems is needed to assess the dynamical implications of instability-driven giant planet migration for the terrestrial planets.

\subsection{Summary}

We have shown that if the terrestrial planets witnessed an epoch when the gross orbital structure of the giant planets changed, then the dynamical structure of the inner solar system was likely altered. In this scenario, the ultimate dynamical state of the terrestrial planets is a product of several processes (e.g., accretion dynamics, passage through secular resonances, AMD diffusion via giant-planet scattering, and chaotic diffusion of the system's AMD over long timescales) that act in concert and whose individual influences are challenging to isolate and disentangle.

The small amplitudes of the $j=2,3$ terrestrial eigenmodes, that are closely associated with the small eccentricities of Earth and Venus, provide strong constraints on the migration of Jupiter and Saturn. While more work examining the characteristic forcing of the terrestrial planets by instability-driven migration is needed, explaining the observed dynamical state of the terrestrial planets as a natural and frequent outcome of the late migration of the giant planets remains a challenge.

An alternate and perhaps simpler possibility is that giant planet formation and migration was largely complete before the dynamical structure of the inner solar system was determined. Formation models and isotopic evidence suggest that terrestrial planet accretion was complete in 30-100 Myr. The bulk of giant planet migration could easily be accommodated in shorter time intervals via some combination of tidal interaction with a gas disk, planet-planet scattering, and planetesimal-driven migration. Any strong perturbation of the inner solar system resulting from giant planet migration might then be tempered through a variety of processes that depend on the state of the forming planets and the protoplanetary disk at the time of the disturbance. Additional mechanisms that may act at this earlier time include dynamical friction with a disk of planetesimals, orbital damping due to interactions with a remnant gas disk, or a combination of dynamical processes (Nagasawa et al. 2005; Thommes et al. 2008a).

In the sequential accretion scenario, the formation timescale for giant planet cores $\left(\sim 10 M_{\oplus}\right)$ in the outer regions of a minimum mass nebula appear to be considerably longer than the observationally inferred depletion timescale of protostellar disks. Ice giant formation at smaller orbital radii (and shorter orbital periods) is often invoked to overcome this problem, but requires the subsequent outward migration of the ice giant planets. However, it is also possible that disk gaps near Jupiter and Saturn's orbits may have provided migration barriers which eventually led to the 
accumulation of planet-building materials and the emergence of Uranus and Neptune within a few million years (see Section 1).

The resonant structure of the Kuiper Belt is often interpreted as a product of planetesimaldriven (Malhotra 1995; Ida et al. 2000; Hahn \& Malhotra 2005) or instability-driven migration (Levison et al. 2008) of the giant planets. However, the giant planets' outward migration may also be induced by their tidal interaction with a viscously expanding gaseous nebula (Lin \& Papaloizou 1986) or by a disk which is undergoing photoevaporation. In these cases, giant planet migration may be mostly complete in $\sim 10 \mathrm{Myr}$ and could predate the final assembly of the terrestrial planets. Such an early migration scenario would avoid the excitation of the terrestrial planets' eccentricities.

This contingency requires that the delivery of LHB impactors to the Moon be achieved after the formation, large-scale migration, and/or global orbital restructuring of the giant planets. Within the context of the Nice model migration scenarios, only explaining the LHB requires that the bulk of giant migration occurred late. If giant planet migration was completed before the dynamical state of the inner solar system was determined, other aspects of the Nice model remain viable (e.g., the capture of Jupiter's Trojans and the orbital properties of the giant planets). Obviously, the delivery of the LHB impactors must then be accounted for via some other means (e.g., the Planet V hypothesis described in Chambers 2007).

Additional progress in unraveling the sequence of events and processes that produced the observed solar system might be made by examining the formation and early evolution of the gas and ice giant planets. We note that the nature and details of gas and ice giant formation in the solar system remain poorly understood. The basic assumptions and initial conditions of current migration models (e.g., that Jupiter and Saturn formed in a much more compact configuration) may change as the formation and early history of the giant planets are clarified.

Finally, we note that migration near and through mean motion resonances is germane to the orbital evolution of planetary systems. Consequently, the resonant sweeping processes examined here have applications among extrasolar planetary systems, satellite systems, and other dynamical characteristics of the solar system. Analyses of these applications will be presented elsewhere.

This work has been supported by NASA (NNG05G1496, NNX07A-L13G, NNX07AI88G, NNX08AL41G, and NNX08AM84G), and the NSF (AST-0908807). We thank Carl Murray and Orkan Umurhan for useful discussions, Tolis Christou for providing the FMFT code used, Ramon Brasser and Alessandro Morbidelli for comments on an early draft of this work, and Renu Malhotra for a thorough and helpful review. 


\section{REFERENCES}

Adams, F. C. \& Laughlin, G. 2006, ApJ, 649, 1004

Agnor, C. B., Canup, R. M., \& Levison, H. F. 1999, Icarus, 142, 219

Agnor, C. B. \& Lin, D. N. 2007, in Bulletin of the American Astronomical Society, 38, 537

Agnor, C. B. \& Ward, W. R. 2002, ApJ, 567, 579

Bailey, B. L. \& Malhotra, R. 2009, Icarus, 203, 155

Batygin, K. \& Brown, M. E. 2010, ApJ, 716, 1323

Bottke, W. F., Vokrouhlicky, D., Nesvorny, D., Minton, D., Morbidelli, A., \& Brasser, R. 2010, Lunar and Planetary Institute Science Conference Abstr., 41, 1269

Brasser, R., Morbidelli, A., Gomes, R., Tsiganis, K., \& Levison, H. F. 2009, A\&A, 507, 1053

Bretagnon, P. 1982, A\&A, 114, 278

Brouwer, D. \& Clemence, G. M. 1961, Methods of Celestial Mechanics (New York: Academic Press, 1961)

Bryden, G., Lin, D. N. C., \& Ida, S. 2000a, ApJ, 544, 481

Bryden, G., Różyczka, M., Lin, D. N. C., \& Bodenheimer, P. 2000b, ApJ, 540, 1091

Chambers, J. E. 2001, Icarus, 152, 205

—. 2007, Icarus, 189, 386

Chambers, J. E. \& Wetherill, G. W. 1998, Icarus, 136, 304

Chiang, E., Lithwick, Y., Murray-Clay, R., Buie, M., Grundy, W., \& Holman, M. 2007, Protostars and Planets V, 895

Christou, A. A. \& Murray, C. D. 1999, MNRAS, 303, 806

Cuk, M. 2007, in Bulletin of the American Astronomical Society, 38, 537

Duncan, M. J., Levison, H. F., \& Lee, M. H. 1998, AJ, 116, 2067

Ellis, K. M. \& Murray, C. D. 2000, Icarus, 147, 129

Evans, N. W. \& Tabachnik, S. A. 2002, MNRAS, 333, L1

Farinella, P., Gonczi, R., Froeschle, C., \& Froeschle, C. 1993, Icarus, 101, 174

Fernandez, J. A. \& Ip, W.-H. 1984, Icarus, 58, 109 
Gladman, B. J., Migliorini, F., Morbidelli, A., Zappala, V., Michel, P., Cellino, A., Froeschle, C., Levison, H. F., Bailey, M., \& Duncan, M. 1997, Science, 277, 197

Goldreich, P., Lithwick, Y., \& Sari, R. 2004, ApJ, 614, 497

Gomes, R., Levison, H. F., Tsiganis, K., \& Morbidelli, A. 2005, Nature, 435, 466

Gomes, R. S. 1997, AJ, 114, 396

Gomes, R. S., Morbidelli, A., \& Levison, H. F. 2004, Icarus, 170, 492

Hahn, J. M. \& Malhotra, R. 1999, AJ, 117, 3041

—. 2005, AJ, 130, 2392

Hansen, B. M. S. 2009, ApJ, 703, 1131

Hartmann, W. K., Ryder, G., Dones, L., \& Grinspoon, D. 2000, in Origin of the Earth and Moon, ed. R.M. Canup and K. Righter, (Tucson: University of Arizona Press), 493

Heppenheimer, T. A. 1980, Icarus, 41, 76

Ida, S., Bryden, G., Lin, D. N. C., \& Tanaka, H. 2000, ApJ, 534, 428

Kleine, T., Münker, C., Mezger, K., \& Palme, H. 2002, Nature, 418, 952

Kretke, K. A. \& Lin, D. N. C. 2007, ApJ, 664, L55

Laskar, J. 1996, Celestial Mechanics and Dynamical Astronomy, 64, 115

-. 2008, Icarus, 196, 1

Levison, H. F., Dones, L., Chapman, C. R., Stern, S. A., Duncan, M. J., \& Zahnle, K. 2001, Icarus, 151,286

Levison, H. F., Lissauer, J. J., \& Duncan, M. J. 1998, AJ, 116, 1998

Levison, H. F. \& Morbidelli, A. 2007, Icarus, 189, 196

Levison, H. F., Morbidelli, A., Gomes, R., \& Backman, D. 2007, Protostars and Planets V, 669

Levison, H. F., Morbidelli, A., Vanlaerhoven, C., Gomes, R., \& Tsiganis, K. 2008, Icarus, 196, 258

Lin, D. N. C., Bodenheimer, P., \& Richardson, D. C. 1996, Nature, 380, 606

Lin, D. N. C. \& Ida, S. 1997, ApJ, 477, 781

Lin, D. N. C. \& Papaloizou, J. 1986, ApJ, 309, 846 
Lissauer, J. J., Pollack, J. B., Wetherill, G. W., \& Stevenson, D. J. 1995, in Neptune and Triton (University of Arizona Press), 37-108

Lissauer, J. J. \& Stevenson, D. J. 2007, Protostars and Planets V, 591

Lyra, W., Paardekooper, S., \& Mac Low, M. 2010, ApJ, 715, L68

Malhotra, R. 1993, Nature, 365, 819

-. 1995, AJ, 110, 420

Malhotra, R., Fox, K., Murray, C. D., \& Nicholson, P. D. 1989, A\&A, 221, 348

Minton, D. A. \& Malhotra, R. 2008, Lunar and Planetary Science Conference Abstr., 39, 2481

Minton, D. A. \& Malhotra, R. 2009, Nature, 457, 1109

—. 2011, ApJ, 732, 53

Morbidelli, A., Brasser, R., Tsiganis, K., Gomes, R., \& Levison, H. F. 2009, A\&A, 507, 1041

Morbidelli, A., Levison, H. F., Tsiganis, K., \& Gomes, R. 2005, Nature, 435, 462

Morbidelli, A., Tsiganis, K., Crida, A., Levison, H. F., \& Gomes, R. 2007, AJ, 134, 1790

Morishima, R., Stadel, J., \& Moore, B. 2010, Icarus, 207, 517

Murray, C. D. \& Dermott, S. F. 1999, Solar System Dynamics (Cambridge University Press)

Murray, N., Hansen, B., Holman, M., \& Tremaine, S. 1998, Science, 279, 69

Nagasawa, M., Lin, D. N. C., \& Thommes, E. 2005, ApJ, 635, 578

O’Brien, D. P., Morbidelli, A., \& Levison, H. F. 2006, Icarus, 184, 39

Papaloizou, J. C. B., Nelson, R. P., Kley, W., Masset, F. S., \& Artymowicz, P. 2007, Protostars and Planets V, 655

Quinn, T. R., Tremaine, S., \& Duncan, M. 1991, AJ, 101, 2287

Rasio, F. A. \& Ford, E. B. 1996, Science, 274, 954

Raymond, S. N., O’Brien, D. P., Morbidelli, A., \& Kaib, N. A. 2009, Icarus, 203, 644

Raymond, S. N., Quinn, T., \& Lunine, J. I. 2004, Icarus, 168, 1

Sidlichovsky, M. \& Nesvorny, D. 1997, Celestial Mechanics and Dynamical Astronomy, 65, 137

Strom, R. G., Malhotra, R., Ito, T., Yoshida, F., \& Kring, D. A. 2005, Science, 309, 1847 
Tera, F., Papanastassiou, D. A., \& Wasserburg, G. J. 1974, Earth and Planetary Science Letters, 22,1

Thommes, E., Nagasawa, M., \& Lin, D. N. C. 2008a, ApJ, 676, 728

Thommes, E. W., Bryden, G., Wu, Y., \& Rasio, F. A. 2008b, ApJ, 675, 1538

Thommes, E. W., Duncan, M. J., \& Levison, H. F. 1999, Nature, 402, 635

—. 2002, AJ, 123, 2862

-. 2003, Icarus, 161, 431

Tiscareno, M. S. \& Malhotra, R. 2003, AJ, 126, 3122

Tsiganis, K., Gomes, R., Morbidelli, A., \& Levison, H. F. 2005, Nature, 435, 459

Walsh, K. J. \& Morbidelli, A. 2011, A\&A, 526, A126+

Ward, W. R. 1981, Icarus, 47, 234

Ward, W. R., Agnor, C. B., \& Tanaka, H. 2001, in ASP Conf. Ser. 245: Astrophysical Ages and Times Scales, ed. T. von Hippel, C. Simpson, \& N. Manset, 111-120

Ward, W. R., Colombo, G., \& Franklin, F. A. 1976, Icarus, 28, 441

Weidenschilling, S. J. 1977, Ap\&SS, 51, 153

Weidenschilling, S. J. \& Marzari, F. 1996, Nature, 384, 619

Wetherill, G. W. 1975, Lunar and Planetary Science Conference Abstr., 6, 866

Yin, Q., Jacobsen, S. B., Yamashita, K., Blichert-Toft, J., Télouk, P., \& Albarède, F. 2002, Nature, 418,949 